\title{
Second-order superintegrable systems in conformally flat spaces. V. Two- and three-dimensional quantum systems
}

\author{
E. G. Kalnins \\ Department of Mathematics and Statistics, University of Waikato, \\ Hamilton, New Zealand \\ J. M. Kress ${ }^{\mathrm{a})}$ \\ School of Mathematics, The University of New South Wales, \\ Sydney NSW 2052, Australia \\ W. Miller, Jr. ${ }^{\text {b) }}$ \\ School of Mathematics, University of Minnesota, Minneapolis, Minnesota, 55455
}

(Received 18 April 2006; accepted 18 July 2006; published online 5 September 2006)

\begin{abstract}
This paper is the conclusion of a series that lays the groundwork for a structure and classification theory of second-order superintegrable systems, both classical and quantum, in conformally flat spaces. For two-dimensional and for conformally flat three-dimensional spaces with nondegenerate potentials we have worked out the structure of the classical systems and shown that the quadratic algebra always closes at order 6 . Here we describe the quantum analogs of these results. We show that, for nondegenerate potentials, each classical system has a unique quantum extension. We also correct an error in an earlier paper in the series (that does not alter the structure results) and we elucidate the distinction between superintegrable systems with bases of functionally linearly independent and functionally linearly dependent symmetries. (C) 2006 American Institute of Physics.
\end{abstract}

[DOI: $10.1063 / 1.2337849]$

\section{INTRODUCTION}

This is the conclusion of a series ${ }^{1-4}$ whose purpose is to lay the groundwork for a structure and classification theory of second-order superintegrable systems, both classical and quantum, in complex conformally flat spaces. Real spaces are considered as restrictions of these to the various real forms. In Refs. 1 and 3 we have given examples in two and three dimensions (2D and 3D), described the background as well as the interest and importance of these systems in mathematical physics, and given dozens of relevant references. Observed features of the systems are multiseparability, closure of the quadratic algebra of second-order symmetries at order 6, use of representation theory of the quadratic algebra to derive spectral properties of the quantum Schrödinger operator, and a close relationship with exactly solvable and quasi-exactly solvable problems. ${ }^{5}$ Our approach is, rather than focus on particular spaces and systems, to use a general theoretical method based on integrability conditions to derive structure common to all systems.

We recall some basic facts and results about conformally flat superintegrable systems. An $n$-dimensional complex Riemannian space is conformally flat if and only if it admits a set of local coordinates $x_{1}, \ldots, x_{n}$ such that the contravariant metric tensor takes the form $g^{i j}=\delta^{i j} / \lambda(\mathbf{x})$. A classical superintegrable system $\mathcal{H}=\sum_{i j} g^{i j} p_{i} p_{j}+V(\mathbf{x})$ on the phase space of this manifold is one that admits $2 n-1$ functionally independent generalized symmetries (or constants of the motion) $\mathcal{S}_{k}, k=1, \ldots, 2 n-1$ with $\mathcal{S}_{1}=\mathcal{H}$ where the $\mathcal{S}_{k}$ are polynomials in the momenta $p_{j}{ }^{6-11}$ It is easy to

\footnotetext{
${ }^{a)}$ Electronic mail: j.kress@unsw.edu.au

${ }^{b)}$ Electronic mail: miller@ima.umn.edu
} 
see that $2 n-1$ is the maximum possible number of functionally independent symmetries and, locally, such (in general nonpolynomial) symmetries always exist. The system is second-order superintegrable if the $2 n-1$ functionally independent symmetries can be chosen to be quadratic in the momenta. Second-order superintegrable systems, though complicated, are tractable because standard orthogonal separation of variables techniques are associated with second-order symmetries, e.g., Refs. 12-17, and these techniques can be brought to bear. Thus we concentrate on second-order superintegrable systems in which the symmetries take the form $\mathcal{S}=\sum a^{i j}(\mathbf{x}) p_{i} p_{j}$ $+W(\mathbf{x})$, quadratic in the momenta.

There is an analogous definition for second-order quantum superintegrable systems with Schrödinger operator

$$
H=\Delta+V(\mathbf{x}), \quad \Delta=\frac{1}{\sqrt{g}} \sum_{i j} \partial_{x_{i}}\left(\sqrt{g} g^{i j}\right) \partial_{x_{j}}
$$

the Laplace-Beltrami operator plus a potential function. ${ }^{12}$ Here there are $2 n-1$ second-order symmetry operators

$$
S_{k}=\frac{1}{\sqrt{g}} \sum_{i j} \partial_{x_{i}}\left(\sqrt{g} a_{(k)}^{i j}\right) \partial_{x_{j}}+W^{(k)}(\mathbf{x}), \quad k=1, \ldots, 2 n-1
$$

with $S_{1}=H$ and $\left[H, S_{k}\right] \equiv H S_{k}-S_{k} H=0$. Again multiseparable systems yield many examples of superintegrability.

The structure theory for classical second-order superintegrable systems with nondegenerate potential for $2 \mathrm{D}$ spaces and for 3D conformally flat spaces has been worked out recently. ${ }^{1-4,18}$ (This paper depends heavily on the results and methods of those papers and we shall refer to them repeatedly.) Each such system has quadratic algebra structure. Let $\left\{\mathcal{S}_{j}\right\}$ be a basis for the secondorder constants of the motion for the Hamiltonian $\mathcal{H}$. By the superintegrability assumption, the Poisson brackets $\left\{\mathcal{S}_{i}, \mathcal{S}_{j}\right\}$ must be functionally dependent on the basis symmetries $\mathcal{S}_{k}$, as are $\left\{\left\{\mathcal{S}_{i}, \mathcal{S}_{j}\right\}, \mathcal{S}_{h}\right\}$ and $\left\{\left\{\mathcal{S}_{i}, \mathcal{S}_{j}\right\},\left\{\mathcal{S}_{h}, \mathcal{S}_{s}\right\}\right\}$. For these systems it is always true that the squares $\left\{\mathcal{S}_{i}, \mathcal{S}_{j}\right\}^{2}$ and products $\left\{\mathcal{S}_{i}, \mathcal{S}_{j}\right\}\left\{\mathcal{S}_{k}, \mathcal{S}_{\ell}\right\}$ as well as $\left\{\left\{\mathcal{S}_{i}, \mathcal{S}_{j}\right\}, \mathcal{S}_{h}\right\}$ and $\left\{\left\{\mathcal{S}_{i}, \mathcal{S}_{j}\right\},\left\{\mathcal{S}_{h}, \mathcal{S}_{s}\right\}\right\}$ are always uniquely expressible as polynomials in the $\left\{\mathcal{S}_{k}\right\}$. This remarkable closure of the algebra generated by the second-order symmetries leads to the very special properties enjoyed by the classical superintegrable systems.

Observed common features of the quantum analogs of these systems are that they are usually multiseparable and that the eigenfunctions of one separable system can be expanded in terms of the eigenfunctions of another. This is the source of nontrivial special function expansion theorems in the quantum case. ${ }^{19}$ The quantum symmetry operators are in formal self-adjoint form and suitable for spectral analysis. Also, the quadratic algebra identities allow us to relate eigenbases and eigenvalues of one symmetry operator to those of another. The representation theory of the abstract quadratic algebra can be used to derive spectral properties of the second-order generators in a manner analogous to the use of Lie algebra representation theory to derive spectral properties of quantum systems that admit Lie symmetry algebras. ${ }^{19-22}$

The structure theory of classical superintegrable systems is simpler than for the quantum case, and we studied it first. However, we now show that each of the classical superintegrable systems with nondegenerate potential has a unique extension to a quantum superintegrable system.

We review, briefly, some basic definitions and notation in the classical 3D case; the corresponding $2 \mathrm{D}$ definitions can be obtained by obvious restriction. For a classical 3D system on a conformally flat space (note that all 2D spaces are conformally flat) we can always choose local coordinates $x, y, z$, not unique, such that the Hamiltonian takes the form $\mathcal{H}=\left(p_{1}^{2}+p_{2}^{2}\right.$ $\left.+p_{3}^{2}\right) / \lambda(x, y, z)+V(x, y, z)$. This system is second-order superintegrable with nondegenerate potential $V=V(x, y, z, \alpha, \beta, \gamma, \delta)$ if it admits five functionally independent quadratic constants of the motion (i.e., generalized symmetries) 


$$
\mathcal{S}_{k}=\sum_{i j} a_{(k)}^{i j} p_{i} p_{j}+W_{(k)}(x, y, \alpha, \beta, \gamma) .
$$

As described in Ref. 3, the potential $V$ is nondegenerate if it satisfies a system of coupled PDEs of the form

$$
\begin{gathered}
V_{22}=V_{11}+A^{22}(x, y, z) V_{1}+B^{22}(x, y, z) V_{2}+C^{22}(x, y, z) V_{3}, \\
V_{33}=V_{11}+A^{33}(x, y, z) V_{1}+B^{33}(x, y, z) V_{2}+C^{33}(x, y, z) V_{3}, \\
V_{12}=A^{12}(x, y, z) V_{1}+B^{12}(x, y, z) V_{2}+C^{12}(x, y, z) V_{3}, \\
V_{13}=A^{13}(x, y, z) V_{1}+B^{13}(x, y, z) V_{2}+C^{13}(x, y, z) V_{3}, \\
V_{23}=A^{23}(x, y, z) V_{1}+B^{23}(x, y, z) V_{2}+C^{23}(x, y, z) V_{3},
\end{gathered}
$$

whose integrability conditions are satisfied identically. Here, $V_{1}=\partial V / \partial x, V_{2}=\partial V / \partial y$, etc. The analytic functions $A^{i j}, B^{i j}, C^{i j}$ are determined uniquely from the Bertrand-Darboux equations for the five constants of the motion (under the assumption that the quadratic constants of the motion are functionally linearly independent) and are analytic except for a finite number of poles. At any regular point $\mathbf{x}_{0}=\left(x_{0}, y_{0}, z_{0}\right)$, i.e., a point where the $A^{i j}, B^{i j}, C^{i j}$ are defined and analytic and the constants of the motion are functionally independent, we can prescribe the values of $V\left(\mathbf{x}_{0}\right), V_{1}\left(\mathbf{x}_{0}\right)$, $V_{2}\left(\mathbf{x}_{0}\right), V_{3}\left(\mathbf{x}_{0}\right), V_{11}\left(\mathbf{x}_{0}\right)$ arbitrarily and obtain a unique solution of (2). The significance of the four parameters for a nondegenerate potential (in addition to the usual additive constant) is that it is the maximum dimension of the space of solutions to the Bertrand-Darboux equations that can appear in a superintegrable system with functionally linearly independent symmetries. If the number of parameters is fewer than four, we say that the superintegrable potential is degenerate.

We clarify our definition of nondegenerate potential and our parameter count by considering the generalized Calogero potential

$$
V^{(1)}=\frac{a}{(x-y)^{2}}+\frac{b}{(y-z)^{2}}+\frac{c}{(z-x)^{2}},
$$

and its further generalization

$$
V^{(2)}=\frac{a}{\left(m_{1} x-m_{2} y\right)^{2}}+\frac{b}{\left(m_{2} y-m_{3} z\right)^{2}}+\frac{c}{\left(m_{3} z-m_{1} x\right)^{2}},
$$

where $m_{i} \neq 0$, see Refs. 11 and 23-25. These potentials are superintegrable on Euclidean space and the second contains six parameters, which exceeds the count of four for nondegenerate superintegrable systems. How can this be?

Our definition of the number of parameters in a superintegrable system is that it is the dimension of the space of solutions of the set of Bertrand-Darboux equations for this system (ignoring the trivial added constant). Let us consider the system of symmetries defining the system with potential $V^{(1)}$. A basis for the space of symmetries is (using $P_{x}=p_{1}, P_{y}=p_{2}, P_{z}=p_{3}, J_{1}=y p_{3}$ $\left.-z p_{2}, J_{2}=z p_{1}-x p_{3}, J_{3}=x p_{2}-y p_{1}\right)$,

$$
\begin{gathered}
\mathcal{S}_{1}=\mathcal{H}=P_{x}^{2}+P_{y}^{2}+P_{z}^{2}+V_{1}, \quad \mathcal{S}_{2}=\left(P_{x}+P_{y}+P_{z}\right)^{2}, \quad \mathcal{S}_{3}=J_{1}^{2}+J_{2}^{2}+J_{3}^{2}+W_{3}, \\
\mathcal{S}_{4}=P_{x}\left(J_{2}-J_{3}\right)+P_{y}\left(J_{3}-J_{1}\right)+P_{z}\left(J_{1}-J_{2}\right)+W_{4}, \quad \mathcal{S}_{5}=J_{3} J_{2}+J_{1} J_{3}+J_{2} J_{1}+W_{5},
\end{gathered}
$$

where the potential terms $W_{i}$ contain the parameters.

We can write the Bertrand-Darboux equations for each symmetry $\mathcal{S}=\sum a^{j k} p_{j} p_{k}+W$ of $\mathcal{H}$ $=\left(p_{1}^{2}+p_{2}^{2}+p_{3}^{3}\right) / \lambda(\mathbf{x})+V$ in the matrix form 


$$
\begin{aligned}
0 & =\left(\begin{array}{ccccc}
0 & a^{12} & a^{11}-a^{22} & a^{31} & -a^{32} \\
a^{13} & 0 & -a^{23} & a^{21} & a^{11}-a^{33} \\
a^{32} & -a^{32} & -a^{13} & a^{22}-a^{33} & a^{12}
\end{array}\right)\left(\begin{array}{c}
V_{33}-V_{11} \\
V_{22}-V_{11} \\
V_{12} \\
V_{32} \\
V_{31}
\end{array}\right)-\frac{1}{\lambda}\left(\begin{array}{c}
\left(\lambda a^{12}\right)_{1}-\left(\lambda a^{11}\right)_{2} \\
\left(\lambda a^{31}\right)_{1}-\left(\lambda a^{11}\right)_{3} \\
\left(\lambda a^{31}\right)_{2}-\left(\lambda a^{21}\right)_{3}
\end{array}\right) V_{1} \\
& -\frac{1}{\lambda}\left(\begin{array}{c}
\left(\lambda a^{22}\right)_{1}-\left(\lambda a^{21}\right)_{2} \\
\left(\lambda a^{32}\right)_{1}-\left(\lambda a^{12}\right)_{3} \\
\left(\lambda a^{32}\right)_{2}-\left(\lambda a^{22}\right)_{3}
\end{array}\right) V_{2}-\frac{1}{\lambda}\left(\begin{array}{c}
\left(\lambda a^{32}\right)_{1}-\left(\lambda a^{31}\right)_{2} \\
\left(\lambda a^{33}\right)_{1}-\left(\lambda a^{13}\right)_{3} \\
\left(\lambda a^{33}\right)_{2}-\left(\lambda a^{23}\right)_{3}
\end{array}\right) V_{3} .
\end{aligned}
$$

In the Euclidean case, $\lambda=1$. Evaluating these equations for potential $V^{(1)}$ we find that they are

$$
\begin{gathered}
V_{1}+V_{2}+V_{3}=0, \quad(x-y) V_{12}+(z-y) V_{23}-V_{1}+2 V_{2}-V_{3}=0, \\
(x-z) V_{13}+(y-z) V_{23}-V_{1}-V_{2}+2 V_{3}=0,
\end{gathered}
$$

and their differential consequences. The complete system of equations is in involution and a particular solution is determined unquely by choosing $V_{2}, V_{3}, V_{23}$ at a regular point. Thus we have a three parameter potential. The apparent six parameter potential $V^{(2)}$ is actually three parameter by our count, because the $m_{i}$ are parametrizing a family of defining symmetries $\mathcal{S}\left(m_{1}, m_{2}, m_{3}\right)$, i.e., the Bertrand-Darboux equations themselves are functions of the $m_{1}$. For example, the symmetry $\mathcal{S}_{2}$ is replaced by $\mathcal{S}_{2}\left(m_{1}, m_{2}, m_{3}\right)=\left(P_{x} / m_{1}+P_{y} / m_{2}+P_{z} / m_{3}\right)^{2}$. Another way to see this is to note that the potentials $V^{(2)}$ do not form a vector space. For each fixed value of the $m_{i}$, i.e., for each fixed choice of the space of defining quadratic symmetries, we have a three parameter potential.

What is important to notice here is the occurrence of the first-order condition $V_{1}+V_{2}+V_{3}=0$ for the potential as a consequence of the Bertrand-Darboux equations. Thus the potential is a function of only two variables, impossible for nondegenerate potentials. To understand this, observe the relation

$$
(x+y+z)^{2} \hat{\mathcal{S}}_{1}-\left(x^{2}+y^{2}+x^{2}\right) \hat{\mathcal{S}}_{2}+2 \hat{\mathcal{S}}_{3}-2(x+y+z) \hat{\mathcal{S}}_{4}-2 \hat{\mathcal{S}}_{5}=0
$$

obeyed by the purely quadratic terms in the symmetries, i.e., where we have set $\mathcal{S}_{i}=\hat{\mathcal{S}}_{i}+W_{i}$. This means that the five functionally independent symmetries $\mathcal{S}_{i}$ are functionally linearly dependent. This dependence reduces the rank of second derivative terms in the system of 12 BertrandDarboux equations so that we do not obtain the canonical form (2) which is required for nondegeneracy. As shown in Ref. 3, if we have a 3D superintegrable system with a basis of functionally linear independent symmetries, then we always obtain the canonical system (2) and its differential consequences.

Functional linear dependence of a functionally independent maximal set of symmetries is hard to achieve. In 2D it is well known that essentially, there is only one example, corresponding to Lie form. In 3D Theorem 1 of Ref. 3 stated, incorrectly, that all functionally independent superintegrable systems were functionally linearly independent. The Calogero potential is a counterexample. Thus the results of papers Refs. 3 and 4 hold under the explicit assumption that the functionally independent basis of symmetries is also functionally linearly independent. This is exactly the same situation as in the $2 \mathrm{D}$ case. ${ }^{1}$

For the following result the system need not be superintegrable.

Theorem 1: Let the functionally independent set $\left\{\mathcal{H}=\mathcal{S}_{1}, \mathcal{S}_{2}, \ldots, \mathcal{S}_{t}\right\},(t>2)$ be a functionally linearly dependent basis of second-order symmetries for the system $\mathcal{H}=\left(p_{1}^{2}+p_{2}^{2}+p_{3}^{3}\right) / \lambda(\mathbf{x})+V$ with nontrivial potential $V$, i.e., there is a relation $\Sigma_{h} c^{(h)}(x) \hat{\mathcal{S}}_{h} \equiv 0$ in an open set, where not all $c^{(h)}$ $\times(x)$ are constants, and no such relation holds for the $c^{(h)}$ all constant, except if the constants are all zero. (Here $\mathcal{S}_{i}=\hat{\mathcal{S}}+W_{i}$ where the $W_{i}$ are the potential terms.) Then the potential must satisfy a first-order relation $A V_{1}+B V_{2}+C V_{3}=0$ where not all of the functions $A, B, C$ are zero.

Proof: By relabeling, we can express one of the quadratic parts of the constants of the motion 
$\hat{\mathcal{S}}_{0}$ as a linear combination of a functionally independent subset $\left\{\hat{\mathcal{S}}_{1}, \ldots, \hat{\mathcal{S}}_{r}, 1 \leqslant r \leqslant 4\right\}$ : $\hat{\mathcal{S}}_{0}$ $=\Sigma_{\ell=1}^{r} c^{(\ell)}(x) \hat{\mathcal{S}}_{\ell}$. Taking the Poisson bracket of both sides of this equation with $\left(p_{1}^{2}+p_{2}^{2}+p_{3}^{3}\right) / \lambda$ and using the fact that each of the $\mathcal{S}_{h}$ is a constant of the motion, we obtain the identity

$$
\sum_{\ell=1}^{r} \sum_{i, j=1}^{3}\left(\partial_{x_{k}} c^{(\ell)}\right) a_{(\ell)}^{i j} p_{i} p_{j} p_{k}=0,
$$

where $(x, y, z) \equiv\left(x_{1}, x_{2}, x_{3}\right)$. It is straightforward to check that this identity can be satisfied if and only if the functions

$$
c_{k}^{i j}=\sum_{\ell=1}^{r}\left(\partial_{x_{k}} c^{(\ell)}\right) a_{(\ell)}^{i j}, \quad 1 \leqslant i, j, k \leqslant 3
$$

satisfy

$$
c_{i}^{i i}=0, \quad c_{j}^{i i}+2 c_{i}^{i j}=0, \quad(i \neq j), \quad c_{3}^{12}+c_{1}^{23}+c_{2}^{31}=0 .
$$

Note that $c_{k}^{i j}=c_{k}^{j i}$. Corresponding to each of the basis symmetries $\mathcal{S}_{h}$ there is a linear set $C_{h}=0$ of Bertrand-Darboux equations (5). A straightforward substitution into the identity $C_{0}-\sum_{\ell=1}^{r} c^{(\ell)}$ $\times(x) C_{\ell}=0$ yields the relation

$$
\left(\begin{array}{c}
c_{1}^{12}-c_{2}^{11} \\
c_{1}^{31}-c_{3}^{11} \\
c_{2}^{31}-c_{3}^{21}
\end{array}\right) V_{1}+\left(\begin{array}{c}
c_{1}^{22}-c_{2}^{21} \\
c_{1}^{32}-c_{3}^{12} \\
c_{2}^{32}-c_{3}^{22}
\end{array}\right) V_{2}+\left(\begin{array}{c}
c_{1}^{32}-c_{2}^{31} \\
c_{1}^{33}-c_{3}^{13} \\
c_{2}^{33}-c_{3}^{23}
\end{array}\right) V_{3}=0
$$

These first-order differential equations for the potential cannot all vanish identically. Indeed if they did all vanish then we would have the conditions

$$
\begin{gathered}
c_{1}^{12}=c_{2}^{11}, \quad c_{1}^{31}=c_{3}^{11}, \quad c_{2}^{31}=c_{3}^{21}, \quad c_{1}^{22}=c_{2}^{21}, \quad c_{1}^{32}=c_{3}^{12}, \\
c_{2}^{32}=c_{3}^{22}, \quad c_{1}^{32}=c_{2}^{31}, \quad c_{1}^{33}=c_{3}^{13}, \quad c_{2}^{33}=c_{3}^{23} .
\end{gathered}
$$

These conditions, together with conditions (8), show that $c_{i}^{j k}=0$ for all $i, j, k$. Thus we have $\sum_{\ell=1}^{r}\left(\partial_{x_{k}} c^{(\ell)}\right) a_{(\ell)}^{i j}=0,1 \leqslant i, j, k \leqslant 3$. Since the set $\left\{\hat{\mathcal{S}}_{1}, \ldots, \hat{\mathcal{S}}_{r}\right\}$, is functionally linearly independent, we have $\partial_{x_{k}} c^{(\ell)} \equiv 0$ for $1 \leqslant k \leqslant 3,1 \leqslant \ell \leqslant r$. Hence the $c^{(\ell)}$ are constants, which means that $\hat{\mathcal{S}}_{0}$ $-\sum_{\ell=1}^{r} c^{(\ell)} \hat{\mathcal{S}}_{\ell}=0$. Thus the set $\left\{\mathcal{S}_{0}, \ldots, \mathcal{S}_{4}\right\}$ is functionally dependent. This is a contradiction.

Q.E.D.

This shows that the potential function for any system, superintegrable or not, with a basis of symmetries that is functionally linearly dependent must satisfy at least one nontrivial first-order partial differential equation $A V_{1}+B V_{2}+C V_{3}=0$ where the functions $A, B, C$ are parameter free. The method of proof of the Theorem shows how to find such equations. This means that all such potentials depend on either one or two coordinates. The 3D nondegenerate potentials that are the primary subject of this series depend essentially on all three coordinates.

\section{NONDEGENERATE 2D QUANTUM SYSTEMS}

Here we discuss how the analysis of classical 2D superintegrable systems with nondegenerate potentials carries over to the quantum case. The quantization is much simpler in the $2 \mathrm{D}$ case than for dimensions greater than two. For a manifold with metric $\mathrm{d} s^{2}=\lambda(x, y)\left(\mathrm{d} x^{2}+\mathrm{d} y^{2}\right)$ the Hamiltonian system $\mathcal{H}=\left(p_{1}^{2}+p_{2}^{2}\right) / \lambda(x, y)+V(x, y)$ is replaced by the Hamiltonian (Schrödinger) operator with potential 


$$
H=\frac{1}{\lambda(x, y)}\left(\partial_{11}+\partial_{22}\right)+V(x, y)
$$

in local orthogonal coordinates. A second-order symmetry of the Hamiltonian system $\mathcal{S}$ $=\Sigma_{k, j=1}^{2} a^{k j}(x, y) p_{k} p_{j}+W(x, y)$, with $a^{k j}=a^{j k}$, corresponds to the operator

$$
S=\frac{1}{\lambda(x, y)} \sum_{k, j=1}^{2} \partial_{k}\left(a^{k j}(x, y) \lambda(x, y) \partial_{j}\right)+W(x, y), \quad a^{k j}=a^{j k} .
$$

These operators are formally self-adjoint with respect to the bilinear product

$$
\langle f, g\rangle=\int f(x, y) g(x, y) \lambda(x, y) \mathrm{d} x \mathrm{~d} y
$$

on the manifold, i.e.,

$$
\langle f, H g\rangle=\langle H f, g\rangle, \quad\langle f, S g\rangle=\langle S f, g\rangle
$$

for all local $C^{\infty}$ functions $f, g$ with compact support on the manifold, where we set all boundary terms equal to 0 .

A first-order symmetry of the Hamiltonian system $\mathcal{L}=\Sigma_{k=1}^{2} a^{k}(x, y) p_{k}$ corresponds to the operator

$$
L=\sum_{k=1}^{2} a^{k}(x, y) \partial_{k} .
$$

It is easy to show that $L_{1}$ is formally skew-adjoint, i.e.,

$$
\langle f, L g\rangle=-\langle L f, g\rangle .
$$

The following results that relate the operator commutator $[A, B]=A B-B A$ and the Poisson bracket are straightforward to verify.

Lemma 1:

$$
\{\mathcal{H}, \mathcal{S}\}=0 \Leftrightarrow[H, S]=0 .
$$

This result is not generally true for higher dimensional manifolds.

Lemma 2:

$$
\{\mathcal{H}, \mathcal{L}\}=0 \Leftrightarrow[H, L]=0 .
$$

The definition of a nondegenerate potential $V(x, y)$ is identical with that for the classical case, i.e., it obeys

$$
\begin{gathered}
V_{22}=V_{11}+A^{22} V_{1}+B^{22} V_{2}, \\
V_{12}=A^{12} V_{1}+B^{12} V_{2}
\end{gathered}
$$

Again, $V_{1}, V_{2}, V_{11}$ can be prescribed arbitrarily at a fixed regular point. Note that if $V$ is a nondegenerate potential then there will be no first-order symmetries.

It follows from Lemma 1 that the classical results for the space of second-order symmetries corresponding to a nondegenerate potential can be taken over without change. The space is three dimensional and at any regular point $\mathbf{x}_{0}$ there exists exactly one symmetry, up to an additive constant, such that $a^{j k}\left(\mathbf{x}_{0}\right)=\alpha^{j k}$ for any constant symmetric matrix $\alpha$.

Now we investigate the space of third-order symmetries, i.e., third-order differential operators $K$ that commute with the Hamiltonian: $[H, K]=0$. In general, determination of the possible operators $K$ is very difficult, but in this case, simplifications make the problem tractable: 
1. We are interested, principally, in the space of third-order symmetries that is spanned by the commutators of second-order symmetries $S$. Since the second-order symmetries are formally selfadjoint, the commutators will be skew-adjoint. Thus we can limit ourselves to $K$ that are skew adjoint.

2. A second reason for considering only skew adjoint $K$ follows from the well-known unique decomposition of a symmetry into a formally skew-adjoint part and a formally self-adjoint part, each of which must itself be a symmetry. Clearly the self-adjoint part of a third-order symmetry must be at most a second-order symmetry, i.e., the third-order terms vanish. For a nondegenerate superintegrable system we already know the three-dimensional space of these second-order symmetries.

3. Since $H$ encodes a three-parameter family of potentials, the symmetry $K$ must also be a function of the parameters. The highest order terms $a^{k j i} \partial_{k j i}$ in $K$ (symmetric in $k, j, i$ ) will be independent of the parameters but lower order terms may have linear parameter dependence.

4. The skew-adjoint requirement uniquely determines the coefficients of the second-order terms in $K$. They are

$$
\frac{3}{2 \lambda}\left(a^{k j i} \lambda\right)_{i} \partial_{k j}
$$

5. Further, the skew-adjoint requirement means that there exist functions $a^{k j i}, \widetilde{b}^{i}$ such that $K$ has the unique representation

$$
K=\sum_{k, j, i=1}^{2}\left(a^{k j i} \partial_{k j i}+\frac{3}{2 \lambda}\left(a^{k j i} \lambda\right)_{i} \partial_{k j}+\frac{1}{2 \lambda}\left(a^{k j i} \lambda\right)_{k j} \partial_{i}\right)+\sum_{i=1}^{2}\left(\tilde{b}^{i} \partial_{i}+\frac{1}{2 \lambda}\left(\tilde{b}^{i} \lambda\right)_{i}\right),
$$

where the functions $\widetilde{b}^{i}(x, y, z)$ contain the parameter dependence.

6. Equating coefficients of the fourth-order terms in the operator condition $[H, K]=0$ where $K$ is given by (11) we obtain the relations

$$
\begin{gathered}
2 \frac{\partial a^{i i i}}{\partial x_{i}}=-3\left(\frac{\partial \ln \lambda}{\partial_{x_{i}}} a^{i i i}+\frac{\partial \ln \lambda}{\partial_{x j}} a^{j i i}\right), \quad i \neq j, \\
3 \frac{\partial a^{j i i}}{\partial x_{i}}+\frac{\partial a^{i i i}}{\partial x_{j}}=3\left(-\frac{\partial \ln \lambda}{\partial_{x_{i}}} a^{i i j}-\frac{\partial \ln \lambda}{\partial_{x_{j}}} a^{i j j}\right), \quad i \neq j, \\
2\left(\frac{\partial a^{122}}{\partial x_{1}}+\frac{\partial a^{112}}{\partial x_{2}}\right)=-\frac{\partial \ln \lambda}{\partial_{x_{1}}} a^{122}-\frac{\partial \ln \lambda}{\partial_{x_{1}}} a^{111}-\frac{\partial \ln \lambda}{\partial_{x_{2}}} a^{222}-\frac{\partial \ln \lambda}{\partial_{x_{2}}} a^{112},
\end{gathered}
$$

which are just the requirements that the $a^{k j i}$ be the components of a third-order Killing tensor.

7. Equating coefficients of the third-order terms in the condition $[H, K]=0$ we obtain relations that are consequences of the Killing tensor relations (12).

8. The remaining conditions on $K$ intertwine $\lambda, a^{k j i}, \widetilde{b}^{i}$, and $V$, and are complicated. Rather than solve them directly, we note that we can make the unique decomposition

$$
\widetilde{b}^{i}\left(x_{1}, x_{2}, V_{x_{1}}, V_{x_{2}}\right)=c^{i}\left(x_{1}, x_{2}\right)+b^{i}\left(x_{1}, x_{2}, V_{x_{1}}, V_{x_{2}}\right),
$$

where 


$$
b^{i}=\sum_{j=1}^{2} f^{\ell, j}\left(x_{1}, x_{2}\right) \frac{\partial V}{\partial x_{j}}\left(x_{1}, x_{2}\right),
$$

i.e., we can split off the parameter-dependent terms of $\widetilde{b}^{i}$ from the rest. Then, equating the linear parameter-dependent coefficients of the second-order terms in the symmetry operator condition, we obtain the requirements

$$
\begin{gathered}
\frac{\partial b^{1}}{\partial x_{2}}+\frac{\partial b^{2}}{\partial x_{1}}=3 \sum_{s=1}^{2} \lambda a^{s 21} \frac{\partial V}{\partial x_{s}}, \\
\frac{\partial b^{j}}{\partial x_{j}}=\frac{3}{2} \sum_{s=1}^{2} a^{s j j} \frac{\partial V}{\partial x_{s}}-\frac{1}{2} \sum_{s=1}^{2} \frac{\partial \ln \lambda}{\partial x_{s}} b^{s}, \quad j=1,2,
\end{gathered}
$$

identical to the corresponding classical equations in Ref. 1. Equating the quadratic parameterdependent coefficients of the zeroth-order terms in the symmetry operator condition, we obtain the requirement

$$
\sum_{s=1}^{2} b^{s} \frac{\partial V}{\partial x_{s}}=0
$$

again identical to the corresponding classical equation in Ref. 1.

9. Conditions (14) and (15), and third-order Killing tensor conditions are clearly necessary for $K$ to be a skew-adjoint symmetry. To see that they are sufficient will take several steps.

10. Uniqueness: Suppose $K, K^{\prime}$ are two third-order skew-adjoint symmetries with the same functions $a^{k j i}, b^{i}$ (but possibly different $c^{i}$ ). Note that $K-K^{\prime}$ is a skew-adjoint, parameterindependent symmetry that is first order (since the third- and second-order terms in $K$ and $K^{\prime}$ are the same). However, there can be no nonzero parameter-independent symmetry for a nondegenerate superintegrable system. Therefore $K=K^{\prime}$. Though we have not given an explicit expression for the $c^{i}$ we see that they are uniquely determined by the functions $a^{k j i}, b^{i}$.

11. Existence: This also involves several steps. We first employ the results of our construction of third-order symmetries for the classical case. There we used (13) to show

$$
f^{\ell, j}+f^{j, \ell}=0, \quad 1 \leqslant \ell, j \leqslant 2,
$$

and (14) to show that

$$
\begin{array}{ll}
b_{1}^{1}=f_{1}^{1,2} V_{2}+f^{1,2} V_{12}, & b_{2}^{1}=f_{2}^{1,2} V_{2}+f^{1,2} V_{22}, \\
b_{1}^{2}=f_{1}^{2,1} V_{1}+f^{2,1} V_{11}, & b_{2}^{2}=f_{2}^{2,1} V_{1}+f^{2,1} V_{12},
\end{array}
$$

and

$$
\begin{gathered}
\lambda a^{111}=\frac{1}{3} f^{1,2}\left(2 A^{12}-(\ln \lambda)_{2}\right), \quad \lambda a^{222}=\frac{1}{3} f^{1,2}\left(-2 B^{12}+(\ln \lambda)_{1}\right), \\
\lambda a^{112}=\frac{1}{9} f^{1,2}\left(2 A^{22}+2 B^{12}+(\ln \lambda)_{1}\right), \\
\lambda a^{122}=\frac{1}{9} f^{1,2}\left(-2 A^{12}+2 B^{22}-(\ln \lambda)_{2}\right), \\
f_{1}^{1,2}=\frac{1}{3} f^{1,2}\left(A^{22}-2 B^{12}-(\ln \lambda)_{1}\right), \quad f_{2}^{1,2}=\frac{1}{3} f^{1,2}\left(-2 A^{12}-B^{22}+(\ln \lambda)_{2}\right) .
\end{gathered}
$$

Thus the $a^{i j k}$ can be expressed in terms of $f^{1,2}$ and the $A^{k \ell}, B^{k \ell}$ functions, and we have an involutive system for $f^{1,2}$. Thus any third symmetry is uniquely determined by the constant 
$f^{1,2}\left(x_{0}, y_{0}\right)$ at some regular point $\left(x_{0}, y_{0}\right)$. This means that the space of third-order skew-adjoint symmetries is at most one dimensional.

12. Consider the case where all $a^{i j k} \equiv 0$. Then $2 A^{12}=B^{22}=(\ln \lambda)_{2}, 2 B^{12}=-A^{22}=(\ln \lambda)_{1}$. The integrability conditions require $(\ln \lambda)_{11}+(\ln \lambda)_{22}=0$, which is the condition for flat space, Thus by an appropriate orthogonal change of coordinates we can assume that $\lambda \equiv 1$. In these new coordinates we see that $A^{i j}=B^{i j} \equiv 0$ for all $i, j$. The general solution is

$$
f^{1,2}=c_{1},
$$

where $c_{1}$, is a constant. This is the homogeneous isotropic oscillator:

$$
V(x, y)=\alpha x+\beta y+\gamma\left(x^{2}+y^{2}\right) .
$$

One can easily check that for this very special case a nonzero commutator of two second-order symmetries is first order, parameter-dependent.

13. The second case is that not all $a^{i j k}$ vanish. We show that the space of symmetries is exactly one dimensional. Let

$$
\left.S_{1}=\frac{1}{\lambda} \sum \partial_{k}\left(a_{(1)}^{k j} \lambda\right) \partial_{j}\right)+W_{(1)}, \quad S_{2}=\sum \partial_{k}\left(a_{(2)}^{k j} \lambda \partial_{j}\right)+W_{(2)}
$$

be second-order symmetries and let $\mathcal{A}_{(i)}\left(x_{1}, x_{2}\right)=\left\{a_{(i)}^{k j}\left(x_{1}, x_{2}\right)\right\}, i=1,2$ be $2 \times 2$ matrix functions. Then the commutator $\left[S_{1}, S_{2}\right]$ of these symmetries is a third-order symmetry $K$ with $a^{k j i}$ and $f^{k, \ell}$ such that

$$
f^{k, \ell}=2 \lambda \sum_{j}\left(a_{(2)}^{k j} a_{(1)}^{j \ell}-a_{(1)}^{k j} a_{(2)}^{j \ell}\right) .
$$

Thus $K=\left[S_{1}, S_{2}\right]$ is uniquely determined by the skew-symmetric matrix

$$
\left[\mathcal{A}_{(2)}, \mathcal{A}_{(1)}\right] \equiv \mathcal{A}_{(2)} \mathcal{A}_{(1)}-\mathcal{A}_{(1)} \mathcal{A}_{(2)},
$$

hence by the constant matrix $\left[\mathcal{A}_{(2)}\left(x_{0}, y_{0}\right), \mathcal{A}_{(1)}\left(x_{0}, y_{0}\right)\right]$ evaluated at a regular point.

Theorem 2: Let $K$ be a third-order skew-adjoint symmetry (11) for a superintegrable system with nondegenerate potential $V$ and $\widetilde{b}^{i}=c^{i}(x, y)+b^{i}\left(x, y, V_{1}, V_{2}\right)$ where

$$
b^{i}=\sum_{j=1}^{2} f^{i, j}(x, y) \frac{\partial V}{\partial x_{j}}(x, y) .
$$

Then

$$
f^{\ell, j}+f^{j, \ell}=0, \quad 1 \leqslant \ell, j \leqslant 2
$$

and $K$ is uniquely determined by the number

$$
f^{1,2}\left(x_{0}, y_{0}\right)
$$

at some regular point $\left(x_{0}, y_{0}\right)$ of $V$.

Corollary 1: Let $V$ be a superintegrable nondegenerate potential, Then the space of thirdorder skew-adjoint symmetries is one dimensional and is spanned by commutators of the secondorder self-adjoint symmetries.

Corollary 2: Let $V$ be a superintegrable nondegenerate potential and $S_{1}, S_{2}$ be second-order formally self-adjoint symmetries with matrices $\mathcal{A}_{(1)}, \mathcal{A}_{(2)}$, respectively. Then

$$
\left[S_{1}, S_{2}\right] \equiv 0 \Leftrightarrow\left[\mathcal{A}_{(1)}, \mathcal{A}_{(2)}\right] \equiv 0 \Leftrightarrow\left[\mathcal{A}_{(1)}\left(\mathbf{x}_{0}\right), \mathcal{A}_{(2)}\left(\mathbf{x}_{0}\right)\right]=0
$$

at a regular point $\mathbf{x}_{0}$. 


\section{A STANDARD FORM FOR 2D QUANTUM SYSTEMS}

In analogy with the classical case, there is a standard structure for $2 \mathrm{D}$ quantum nondegenerate superintegrable systems allowing the identification of the space of second-order symmetry operators with the space of $2 \times 2$ symmetric matrices, and identification of the space of third-order symmetry operators with the space of $2 \times 2$ skew-symmetric matrices. Indeed, if $\mathbf{x}_{0}$ is a regular point then there is a 1-1 linear correspondence between second-order operators $S$ and their associated symmetric matrices $\mathcal{A}\left(\mathbf{x}_{0}\right)$. Let $\left[S_{1}, L_{2}\right]^{\prime}=\left[S_{2}, S_{1}\right]$ be the reversed operator commutator. Then the map

$$
\left[S_{1}, S_{2}\right]^{\prime} \leftrightarrow\left[\mathcal{A}_{(1)}\left(\mathbf{x}_{0}\right), \mathcal{A}_{(2)}\left(\mathbf{x}_{0}\right)\right]
$$

is an algebraic isomorphism. Here, $S_{1}, S_{2}$ are in involution if and only if matrices $\mathcal{A}_{(1)}\left(\mathbf{x}_{0}\right), \mathcal{A}_{(2)}$ $\times\left(\mathbf{x}_{0}\right)$ commute. If $\left[S_{1}, S_{2}\right] \neq 0$ then it is a truly third-order symmetry operator (except in the isotropic oscillator case) and can be uniquely associated with the skew-symmetric matrix $\left[\mathcal{A}_{(1)}\right.$ $\times\left(\mathbf{x}_{0}\right), \mathcal{A}_{(2)}\left(\mathbf{x}_{0}\right)$ ]. Since commutators of second-order symmetries span the space of third-order symmetries, we can identify these $1-1$ with $2 \times 2$ skew-symmetric matrices. Let $\mathcal{E}^{i j}$ be the $2 \times 2$ matrix with a 1 in row $i$, column $j$ and 0 for every other matrix element. Then the symmetric matrices

$$
\mathcal{A}^{(i j)}=\frac{1}{2}\left(\mathcal{E}^{i j}+\mathcal{E}^{j i}\right)=\mathcal{A}^{(j i)}, \quad i, j=1,2
$$

form a basis for the three-dimensional space of symmetric matrices. Moreover,

$$
\left[\mathcal{A}^{(i j)}, \mathcal{A}^{(k \ell)}\right]=\frac{1}{2}\left(\delta_{j k} \mathcal{B}^{(i \ell)}+\delta_{j \ell} \mathcal{B}^{(i k)}+\delta_{i k} \mathcal{B}^{(j \ell)}+\delta_{i \ell} \mathcal{B}^{(j k)}\right)
$$

where

$$
\mathcal{B}^{(i j)}=\frac{1}{2}\left(\mathcal{E}^{i j}-\mathcal{E}^{j i}\right)=-\mathcal{B}^{(j i)}, \quad i, j=1,2 .
$$

Here $\mathcal{B}^{(i i)}=0$ and $\mathcal{B}^{(12)}$ forms a basis for the space of skew-symmetric matrices. Thus (17) gives the commutation relations for the second-order symmetry operators. If $V$ is the isotropic oscillator then there is no truly third-order symmetry. For any other nondegenerate potential, the space of symmetries is exactly one dimensional.

We reformulate the problem of determining the second-order symmetry operators of (9) by setting

$$
W(x)=f^{1} V_{1}+f^{2} V_{2}+f^{11} V_{11}
$$

and substituting this expression into $W_{i}=\lambda \sum_{j=1}^{2} a^{i j} V_{j}$. Additionally we must impose the Killing tensor conditions. We obtain the equations for the $a^{i j}$ :

$$
\begin{aligned}
& \lambda a^{11}=f_{1}^{1}+f^{2} A^{12}+f^{11} A^{13}, \\
& \lambda a^{12}=f_{2}^{1}+f^{1} A^{12}+f^{2} A^{22}, \\
& \lambda a^{22}=f_{2}^{2}+f^{1} B^{12}+f^{2} B^{22},
\end{aligned}
$$

and the condition on the first derivatives of the $f^{i}$ :

$$
f_{2}^{1}-f_{1}^{2}=-f^{1} A^{12}+f^{2}\left(A^{22}-B^{12}\right)-f^{11} B^{13} .
$$

Note the expressions for $f_{1}^{11}$ and $f_{2}^{11}$ in terms of $f^{1}, f^{2}, f^{11}$ :

$$
f_{1}^{11}+f^{1}+f^{11}\left(B^{12}-A^{22}\right)=0, \quad f_{2}^{11}+f^{2}+f^{11} A^{12}=0 .
$$

It follows that we can express each of the second derivatives of $f^{1}, f^{2}$ in terms of lower order derivatives of $f^{1}, f^{2}, f^{11}$. Thus the system is in involution at the second derivative level, but not at 
the first derivative level because we have only one condition for the four derivatives $f_{1}^{1}, f_{2}^{1}, f_{1}^{2}, f_{2}^{2}$. We can uniquely determine a symmetry operator at a regular point by choosing the six parameters $\left(f^{1}, f^{2}, f^{11}, f_{1}^{1}, f_{2}^{1}, f_{2}^{2}\right)$. The values of $f^{1}, f^{2}, f^{11}$ at the regular point are analogous to the three parameters that we can add to the potentials in the three parameter family. For our standard basis, we fix $\left(f^{1}, f^{2}, f^{11}\right)_{\mathbf{x}_{0}}=(0,0,0)$. Then from (18) and (19), we have

$$
\left(\begin{array}{ll}
f_{1}^{1} & f_{2}^{1} \\
f_{1}^{2} & f_{2}^{2}
\end{array}\right)=\lambda\left(\begin{array}{ll}
a^{11} & a^{12} \\
a^{21} & a^{22}
\end{array}\right) .
$$

Thus we can define a standard set of basis symmetry operators $S^{(j k)}=\lambda^{-1}(\mathbf{x})\left(\sum \partial_{i}\left(a^{i j}(\mathbf{x}) \lambda(\mathbf{x}) \partial_{j}\right)\right.$ $+W^{(i j)}(\mathbf{x})$ corresponding to a regular point $\mathbf{x}_{0}$ by

$$
\left(\begin{array}{ll}
f_{1}^{1} & f_{2}^{1} \\
f_{1}^{2} & f_{2}^{2}
\end{array}\right)_{\mathbf{x}_{0}}=\lambda\left(\mathbf{x}_{0}\right)\left(\begin{array}{ll}
a^{11} & a^{12} \\
a^{21} & a^{22}
\end{array}\right)_{\mathbf{x}_{0}}=\lambda\left(\mathbf{x}_{0}\right) \mathcal{A}^{(j k)}, \quad W^{(j k)}\left(\mathbf{x}_{0}\right)=0 .
$$

The condition on $W^{(j k)}$ is actually three conditions since $W^{(j k)}$ depends on three parameters. Note that the derivative terms $a_{i}^{i j}$ in the expression for the basis symmetries can be computed explicitly from the conditions for classical second order symmetries in Ref. 1.

In exact analogy with the classical case, we can use the standard form to prove multiseparabilty for quantum systems.

Theorem 3. Let $V$ be a quantum superintegrable nondegenerate potential and $S$ be a secondorder symmetry operator with matrix function $\mathcal{A}(\mathbf{x})$. If at some regular point $\mathbf{x}_{0}$ the matrix $\mathcal{A}\left(\mathbf{x}_{0}\right)$ has two distinct eigenvalues, then $H, S$ characterize an orthogonal separable coordinate system.

Since a generic $2 \times 2$ symmetric matrix has distinct roots, it follows that any such superintegrable nondegenerate potential is multiseparable.

\section{THE QUANTUM QUADRATIC ALGEBRA}

We investigate the space of fourth-order differential operators $F$ that commute with the Hamiltonian: $[H, F]=0$. Determination of all possible operators $F$ is very difficult but, again, there are simplifications that make the problem tractable:

1. We are interested, principally, in the space of fourth-order symmetries that is spanned by the double commutators $\left[\left[S^{(1)}, S^{(2)}\right], S^{(3)}\right]$ of second-order formally self-adjoint symmetries $S^{(j)}$ of the superintegrable system. The double commutators will be formally self-adjoint, so we can limit ourselves to $F$ that are self-adjoint.

2. Since $H$ encodes a three-parameter family of potentials, the symmetry $F$ must also be a function of the parameters. The highest order terms $a^{\ell k j i} \partial_{k j i}$ in $F$ (symmetric in $\ell, k, j, i$ ) will be independent of the parameters but lower order terms may have linear or quadratic parameter dependence.

3. The self-adjoint requirement uniquely determines the third-order terms in $F$. They are

$$
\sum_{\ell, k, j, i} \frac{2}{\lambda}\left(a^{\ell k j i} \lambda\right)_{i} \partial_{\ell k j}
$$

4. Further, the self-adjoint requirement means that there exist functions $a^{\ell k j i}, \widetilde{b}^{i j}, \widetilde{W}$ such that $F$ has the unique representation

$$
F=\sum_{\ell, k, j, i=1}^{2} \frac{1}{\lambda} \partial_{i j}\left(a^{\ell k j i} \lambda \partial_{k \ell}\right)+\sum_{i, j=1}^{2} \frac{1}{\lambda} \partial_{i}\left(\tilde{b}^{i j} \lambda \partial_{j}\right)+\widetilde{W},
$$

where the functions $\tilde{b}^{i j}\left(x_{1}, x_{2}\right), \widetilde{W}\left(x_{1}, x_{2}\right)$ contain the parameter dependence.

5. Equating coefficients of the fifth-order terms in the operator condition $[H, F]=0$ where $F$ is given by (20) we obtain the relations 


$$
\begin{gathered}
\frac{\partial a^{i i i i}}{\partial x_{i}}=-2 \sum_{s=1}^{2} a^{s i i i} \frac{\partial \ln \lambda}{\partial x_{s}}, \\
4 \frac{\partial a^{j i i i}}{\partial x_{i}}+\frac{\partial a^{i i i i}}{\partial x_{j}}=-6 \sum_{s=1}^{2} a^{s i i j} \frac{\partial \ln \lambda}{\partial x_{s}}, \quad i \neq j, \\
3 \frac{\partial a^{j j i i}}{\partial x_{i}}+2 \frac{\partial a^{i i i j}}{\partial x_{j}}=-\sum_{s=1}^{2} a^{s i i i} \frac{\partial \ln \lambda}{\partial x_{s}}-3 \sum_{s=1}^{2} a^{s i j j} \frac{\partial \ln \lambda}{\partial x_{s}}, \quad i \neq j,
\end{gathered}
$$

which are the conditions for $a^{\ell k j i}$ to be a fourth-order Killing tensor.

6. The remaining conditions on $F$ intertwine $\lambda, a^{\ell k j i}, \widetilde{b}^{j i}, \widetilde{W}$, and $V$, and are complicated. Rather than solve them directly, we make the unique decomposition

$$
\tilde{b}^{j i}\left(x_{1}, x_{2}, V_{x_{1}}, V_{x_{2}}, V_{x_{1} x_{1}}\right)=c^{j i}\left(x_{1}, x_{2}\right)+b^{j i}\left(x_{1}, x_{2}, V_{x_{1}}, V_{x_{2}}, V_{x_{1} x_{1}}\right),
$$

where

$$
b^{j i}=\sum_{\alpha=1}^{3} f^{j i, \alpha} W^{(\alpha)}, \quad f^{j i, \alpha}=f^{i j, \alpha},
$$

and $W^{(\alpha)}$ is defined by

$$
\left(\begin{array}{l}
W^{(1)} \\
W^{(2)} \\
W^{(3)}
\end{array}\right)=\left(\begin{array}{c}
V_{x_{1}} \\
V_{x_{2}} \\
V_{x_{1} x_{1}}
\end{array}\right) .
$$

Then, equating the linear parameter-dependent terms of third order in the derivatives we obtain the conditions

$$
\begin{aligned}
\frac{\partial}{\partial x_{h}} f^{j k, \alpha}+\frac{\partial}{\partial x_{k}} f^{h j, \alpha}+\frac{\partial}{\partial x_{j}} f^{k h, \alpha}-2 \lambda a^{\alpha h j k}= & -\sum_{\gamma=1}^{3}\left(f^{j k, \gamma} A_{\gamma \alpha}^{(h)}+f^{h j, \gamma} A_{\gamma \alpha}^{(k)}+f^{k h, \gamma} A_{\gamma \alpha}^{(j)}\right) \\
& -\sum_{s=1}^{2}\left(f^{s k, \alpha} \delta_{h k}+f^{s j, \alpha} \delta_{k h}+f^{s h, \alpha} \delta_{j k}\right) \frac{\partial}{\partial x_{s}} \ln \lambda,
\end{aligned}
$$

where $1 \leqslant j, k, h \leqslant 2$ and we set $a^{3 h j k} \equiv 0$. These conditions are identical to the corresponding classical conditions in Ref. 1. Similarly, we set

$$
\widetilde{W}=U^{(0)}\left(x_{1}, x_{2}\right)+U^{(1)}\left(x_{1}, x_{2}, W^{(\alpha)}\right)+W\left(x_{1}, x_{2}, W^{(\alpha)}\right),
$$

where $U^{(1)}$ depends linearly and $W$ depends quadratically on the $W^{(\alpha)}$ and equate the quadratic parameter-dependent terms of first order in the derivatives. We obtain the conditions

$$
\lambda \sum_{s=1}^{3} b^{s i} \frac{\partial V}{\partial x_{s}}=\frac{\partial W}{\partial x_{i}} .
$$

Equating the quadratic parameter-dependent coefficients of the zeroth-order terms in the symmetry operator condition, we obtain the requirement 


$$
\sum_{s=1}^{2} b^{s} \frac{\partial V}{\partial x_{s}}=0
$$

identical to (13). From the integrabilty conditions $\left(\partial / \partial x_{j}\right)\left(\partial W / \partial x_{i}\right)=\left(\partial / \partial x_{i}\right)\left(\partial W / \partial x_{j}\right), i \neq j$ for Eq. (23) we obtain the conditions

$$
\begin{aligned}
\partial_{x_{j}} f^{\beta k, \alpha}+\partial_{x_{j}} f^{\alpha k, \beta}-\partial_{x_{k}} f^{\beta j, \alpha}-\partial_{x_{k}} f^{\alpha j, \beta}= & \sum_{s=1}^{2}\left(A_{\beta s}^{(k)} f^{s j, \alpha}+A_{\alpha s}^{(k)} f^{s j, \beta}-A_{\beta s}^{(j)} f^{s k, \alpha}-A_{\alpha s}^{(j)} s^{s k, \beta}\right)+\sum_{\gamma=1}^{3}\left(f^{\beta j, \gamma} A_{\gamma \alpha}^{(k)}\right. \\
& \left.+f^{\alpha j, \gamma} A_{\gamma \beta}^{(k)}-f^{\beta k, \gamma} A_{\gamma \alpha}^{(j)}-f^{\alpha k, \gamma} A_{\gamma \beta}^{(j)}\right)-\left(f^{\beta k, \alpha}+f^{\alpha k, \beta}\right) \frac{\partial}{\partial x_{j}} \ln \lambda \\
& +\left(f^{\beta j, \alpha}+f^{\alpha j, \beta}\right) \frac{\partial}{\partial x_{k}} \ln \lambda
\end{aligned}
$$

where $j \neq k, 1 \leqslant \alpha, \beta \leqslant 3$, and we set $f^{3 j, \alpha} \equiv 0$.

7. There are eight independent equations (22) with $\alpha \neq 3$ and we use five of these to define the five components $a^{i h j k}$ as linear combinations of $\left(\partial / \partial x_{h}\right) f^{j k, \alpha}$ and $f^{j k, \alpha}$. We can then eliminate the $a^{i h j k}$ from the remaining three equations to obtain three conditions relating $\left(\partial / \partial x_{h}\right) f^{j k, \alpha}$ and $f^{j k, \alpha}$. There are six terms of the form $\left(\partial / \partial x_{h}\right) f^{j k, 3}$. Equation (25) with $\alpha=\beta=3$ is satisfied identically. There are two equations (25) with $\beta=3,1 \leqslant \alpha \leqslant 2$ and four equations (22) with $\alpha=3$. Thus all six terms of the form $\left(\partial / \partial x_{h}\right) f^{j k, 3}$ can be expressed as linear combinations of $f^{j k, \alpha}$. There are a total of twelve distinct terms of the form $\left(\partial / \partial x_{h}\right) f^{j k, m}, 1 \leqslant h, j, k, m \leqslant 2$. We have seen that there are three conditions on these terms remaining from (22); there are an additional three such conditions from (25) with $\alpha, \beta \neq 3$. Thus there is a shortfall of six conditions on the first derivatives $\left(\partial / \partial x_{h}\right) f^{j k, m}$.

8. There are a total of eighteen distinct terms of the form $\left(\partial^{2} / \partial x_{h} \partial x_{\ell}\right) f^{j k, m}$ with 1 $\leqslant h, j, k, \ell, m \leqslant 2$. Differentiating with respect to $x_{1}, x_{2}$ the three first-order conditions of (22), from which the $a^{i h j k}$ have been eliminated, we obtain six independent conditions on these second derivatives. Differentiating each of our expressions for the $a^{i h j k}$ and substituting into Eq. (21) we find six additional conditions on the second derivatives. Also, we can differentiate the three equations from (23) with $\alpha, \beta \neq 3$ to obtain six additional conditions on the second derivatives. This allows us to express each second-order derivative as a linear combination of lower order derivatives. Thus the system is in involution.

9. Conditions (22) and (23), and the fourth-order Killing tensor conditions are clearly necessary for $F$ to be a skew-adjoint symmetry. To see that they are sufficient will take several steps.

10. Suppose $F, F^{\prime}$ are two fourth-order self-adjoint symmetries with the same functions $a^{\ell k j i}, b^{i j}, W$ (but possibly different $\left.c^{i j}, U^{(j)}\right)$. Then $F-F^{\prime}$ is a self-adjoint, symmetry that is second order and at most linear in the parameters in the zeroth-order term. Thus the only ambiguity is a second-order symmetry operator and we already know these.

11. We conclude that any (truly) fourth-order symmetry operator is uniquely determined, up to an additive second-order symmetry operator, by the values $f^{j k, \alpha}\left(\mathbf{x}_{0}\right)$ and a subset of six of the values $\left(\partial / \partial_{x_{h}}\right) f^{j k, m}\left(\mathbf{x}_{0}\right)$ at a regular point $\mathbf{x}_{0}$. Note that by adding an appropriate linear combination of purely second-order symmetry operators to the fourth-order symmetry we can achieve $f^{j k, \alpha}\left(\mathbf{x}_{0}\right)=0$ for all $j, k, \alpha$, so the maximum possible dimension of the space of purely fourth-order symmetries is six.

Now any symmetric second-order polynomial in the second-order symmetry operators is a fourth-order symmetry operator, and the subspace of polynomial symmetries is at least five and at most six. We show that it is exactly six. If $A, B$ are linear operators, we define their symmetrized product by

$$
\{A, B\} \equiv \frac{1}{2}(A B+B A) .
$$

Theorem 4: The six distinct monomials 


$$
\left\{S^{(11)}, S^{(11)}\right\}, \quad\left\{S^{(22)}, S^{(22)}\right\}, \quad\left\{S^{(12)}, S^{(12)}\right\}, \quad\left\{S^{(11)}, S^{(22)}\right\}, \quad\left\{S^{(11)}, S^{(12)}\right\}, \quad\left\{S^{(12)}, S^{(22)}\right\},
$$

form a basis for the space of fourth-order symmetry operators.

Proof: Since the second-order parts of the three symmetry operators $S^{(11)}, S^{(22)}, S^{(12)}$ are functionally independent, the six monomials listed above are linearly independent. Hence they form a basis.

Q.E.D.

We can use this result to expand explicitly a general fourth-order self-adjoint symmetry

$$
F=\sum_{\ell, k, j, i=1}^{2} \frac{1}{\lambda} \partial_{i j}\left(a^{\ell k j i} \lambda \partial_{k \ell}\right)+\sum_{i, j=1}^{2} \frac{1}{\lambda} \partial_{i}\left(\tilde{b}^{i j} \lambda \partial_{j}\right)+\tilde{W}
$$

in terms of the standard basis. Without loss of generality we can assume that $(0,0)=\mathbf{0}$ is a regular point. Then $F$ is uniquely determined (up to an additive second-order self-adjoint symmetry) by the data $a^{\ell k j i}(\mathbf{0}), \partial_{m} a^{\ell k j i}(\mathbf{0}), b^{m q}(\mathbf{0}), W(\mathbf{0})$. We can uniquely match the data $a^{\ell k j i}(\mathbf{0})$ by taking a linear combination of the basis symmetries

$$
\left\{S^{(11)}, S^{(11)}\right\}, \quad\left\{S^{(22)}, S^{(22)}\right\}, \quad\left\{S^{(12)}, S^{(12)}\right\}, \quad\left\{S^{(11)}, S^{(12)}\right\}, \quad\left\{S^{(12)}, S^{(22)}\right\}, \quad\left\{S^{(11)}, S^{(11)}\right\} .
$$

This leaves the symmetry $\left\{S^{(11)}, S^{(22)}\right\}-\left\{S^{(12)}, S^{(12)}\right\}$ whose leading order terms vanish at the regular point. The expansion coefficient for this term is obtained uniquely from the derivative data $\partial_{m} a^{\ell k j i}(\mathbf{0})$. Now we have matched all of the fourth-order terms in $F$ with an expansion of the self-adjoint form $F^{\prime}=\sum \xi_{i j k \ell}\left\{S^{(i j)}, S^{(k \ell)}\right\}$. The difference $F-F^{\prime}$ is a second-order self-adjoint symmetry. The second derivative terms are uniquely determined by the data $b^{m q}(\mathbf{0}), W(\mathbf{0})$, which has not changed since $W^{(i j)}(\mathbf{0})=0$ for all terms in the standard basis, by the data $\partial_{m} a^{\ell k j i}(\mathbf{0}), \partial_{m s} a^{\ell k j i}(\mathbf{0})$, and by the coefficients $\xi_{i j k \ell}$ which have changed. Thus we can expand the original symmetry in terms of second-order polynomials in the standard basis, and finally add any constant parameterdependent terms. In contrast to the classical case, however, this expansion is more complicated because the expansion coefficients at the fourth-order level effect the expansion coefficients at the second-order level

Using an approach very similar to the above we can easily show that the space of truly sixth-order formally self-adjoint operator symmetries of $H$ cannot exceed the classical maximal dimension of ten. The difference between any two such sixth-order symmetries with the same classical data will be a formally self-adjoint fourth-order symmetry. It remains to show that the maximum possible dimension is actually achieved. If $A, B, C$ are linear operators, we define their symmetrized product by

$$
\{A, B, C\} \equiv \frac{1}{6}(A B C+B A C+C A B+A C B+B C A+C B A) .
$$

Theorem 5: The ten distinct monomials

$$
\begin{gathered}
\left\{S^{(i i)}, S^{(i i)}, \quad S^{(i i)}\right\}, \quad\left\{S^{(i j)}, S^{(i j)}, S^{(i j)}\right\}, \quad\left\{S^{(i i)}, S^{(i i)}, S^{(i j)}\right\}, \\
\left\{S^{(i j)}, S^{(i j)}, S^{(i i)}\right\}, \quad\left\{S^{(11)}, S^{(12)}, S^{(22)}\right\},
\end{gathered}
$$

for $i, j=1,2, i \neq j$ form a basis for the space of sixth-order symmetries.

Proof: Since the three symmetries $S^{(11)}, S^{(22)}, S^{(12)}$ have functionally independent second-order terms, the ten monomials listed above are linearly independent. Hence they form a basis. Q.E.D.

These theorems establish the closure of the quadratic algebra for 2D quantum superintegrable potentials: All fourth-order and sixth-order symmetry operators can be expressed as symmetric polynomials in the second-order symmetry operators.

Again, we can use these results to expand explicitly a general sixth-order formally self-adjoint symmetry operator 


$$
G=\sum_{n, m, \ell, k, j, i=1}^{2} \frac{1}{\lambda} \partial_{n m \ell}\left(a^{n m \ell k j i} \lambda \partial_{k j i}\right)+\sum_{\ell, k, j, i=1}^{2} \frac{1}{\lambda} \partial_{i j}\left(\tilde{b}^{\ell k j i} \lambda \partial_{k \ell}\right)+\sum_{i, j=1}^{2} \frac{1}{\lambda} \partial_{i}\left(\tilde{c}^{i j} \lambda \partial_{j}\right)+\tilde{W}
$$

in terms of the standard symmetrized basis. Here $\tilde{b}^{\ell k j i}, \tilde{c}^{i j}, \widetilde{W}$ are at most linear, quadratic, and cubic in the parameters of the potential, respectively. Without loss of generality we can assume that $(0,0)=\mathbf{0}$ is a regular point. We can uniquely match the data $a^{i j k l m n}(\mathbf{0})$ by taking a linear combination of the seven symmetries

$$
\left\{S^{(i i)}, S^{(i i)}, S^{(i i)}\right\}, \quad\left\{S^{(i j)}, S^{(i j)}, S^{(i j)}\right\}, \quad\left\{S^{(i i)}, S^{(i i)}, S^{(j j)}\right\}, \quad\left\{S^{(i i)}, S^{(i i)}, S^{(i j)}\right\},
$$

for $i, j=1,2, i \neq j$. This leaves the three symmetries

$$
\begin{gathered}
\left\{S^{(11)}, S^{(11)}, S^{(22)}\right\}-\left\{S^{(11)}, S^{(12)}, S^{(12)}\right\}, \quad\left\{S^{(12)}, S^{(11)}, S^{(22)}\right\}-\left\{S^{(12)}, S^{(12)}, S^{(12)}\right\}, \\
\left\{S^{(22)}, S^{(22)}, S^{(11)}\right\}-\left\{S^{(22)}, S^{(12)}, S^{(12)}\right\},
\end{gathered}
$$

whose leading order terms vanish at the regular point. The expansion coefficients for these three terms are obtained uniquely from the derivative data $\partial_{q} a^{i j k l m n}$. Now we have matched all of the sixth-order terms in $G$ with a self-adjoint expansion of the form $G^{\prime}=\sum \xi_{i j k l m n}\left\{S^{(i j)}, S^{(k l)}, S^{(m n)}\right\}$. The difference $G-G^{\prime}$ is a fourth-order self-adjoint symmetry. It is uniquely determined by the data for the even order terms of $G$ and by the new data for the even order terms of $G^{\prime}$. Now we can use the above-presented argument to expand this fourth-order symmetry in terms of polynomials in the standard basis. The expansion coefficients $\xi_{i j k l m n}$ will be the same as for the classical case, but the lower order expansion coefficients will differ.

\section{THE STÄCKEL TRANSFORM FOR 2D QUANTUM SYSTEMS}

The quantum analog of the Stäckel transform ${ }^{26}$ or coupling constant metamorphosis ${ }^{27}$ for classical systems is straightforward in the $2 \mathrm{D}$ case. Suppose we have a superintegrable system

$$
H=\frac{1}{\lambda(x, y)}\left(\partial_{11}+\partial_{22}\right)+V(x, y)=H_{0}+V
$$

in local orthogonal coordinates, with nondegenerate potential $V(x, y)$ :

$$
\begin{gathered}
V_{22}=V_{11}+A^{22} V_{1}+B^{22} V_{2}, \\
V_{12}=A^{12} V_{1}+B^{12} V_{2}
\end{gathered}
$$

and suppose $U(x, y)$ is a particular solution of Eq. (27), nonzero in an open set. Then the transformed system

$$
\widetilde{H}=\frac{1}{\tilde{\lambda}(x, y)}\left(\partial_{11}+\partial_{22}\right)+\tilde{V}(x, y)
$$

with nondegenerate potential $\widetilde{V}(x, y)$ :

$$
\begin{gathered}
\tilde{V}_{22}=\widetilde{V}_{11}+\widetilde{A}^{22} \widetilde{V}_{1}+\widetilde{B}^{22} \widetilde{V}_{2} \\
\tilde{V}_{12}=\widetilde{A}^{12} \widetilde{V}_{1}+\widetilde{B}^{12} \widetilde{V}_{2}
\end{gathered}
$$

is also superintegrable, where

$$
\tilde{\lambda}=\lambda U, \quad \widetilde{V}=\frac{V}{U},
$$




$$
\widetilde{A}^{12}=A^{12}-\frac{U_{2}}{U}, \quad \widetilde{A}^{22}=A^{22}+2 \frac{U_{1}}{U}, \quad \widetilde{B}^{12}=B^{12}-\frac{U_{1}}{U}, \quad \widetilde{B}^{22}=B^{22}-2 \frac{U_{2}}{U} .
$$

Indeed, let $S=\Sigma 1 / \lambda \partial_{i}\left(a^{i j} \lambda \partial_{j}\right)+W=S_{0}+W$ be a second-order formally self-adjoint symmetry operator of $H$ and $S_{U}=\Sigma 1 / \lambda \partial_{i}\left(a^{i j} \lambda \partial_{j}\right)+W_{U}=S_{0}+W_{U}$ be the special case of this that is in involution with $(1 / \lambda)\left(\partial_{11}+\partial_{22}\right)+U$. Then

$$
\widetilde{S}=S_{0}-\frac{W_{U}}{U} H+\frac{1}{U} H
$$

is the corresponding formally self-adjoint symmetry operator of $\widetilde{H}$, with respect to the metric $\mathrm{d} \widetilde{s}^{2}=\lambda U\left(\mathrm{~d} x^{2}+\mathrm{d} y^{2}\right)$.

Theorem 6:

1.

$$
[\tilde{H}, \tilde{S}]=0 \Leftrightarrow[H, S]=0 .
$$

2.

$$
\tilde{S}=\sum_{i j} \frac{1}{\lambda U} \partial_{i}\left(\left(a^{i j}+\delta^{i j} \frac{1-W_{U}}{\lambda U}\right) \lambda U\right) \partial_{j}+\left(W-\frac{W_{U} V}{U}+\frac{V}{U}\right) .
$$

Proof:

1. This is a straightforward verification, using the identities

$$
\left[H_{0}, S_{0}\right]=0, \quad\left[H_{0}+V, S_{0}+W\right]=0, \quad\left[H_{0}+U, S_{0}+W_{U}\right]=0
$$

and

$$
[A, B C]=B[A, C]+[A, B] C, \quad\left[A, \frac{1}{U}\right]=-\frac{1}{U}[A, U] \frac{1}{U}
$$

for linear operators $A, B, C$ and nonzero function $U$.

2. This follows from the fact that $\partial_{i} W_{U}=\lambda \Sigma_{j} a^{i j} U_{j}$.

Corollary 3: If $S^{(1)}, S^{(2)}$ are second-order symmetry operators for $H$, then

$$
\left[\widetilde{S}^{(1)}, \widetilde{S}^{(2)}\right]=0 \Leftrightarrow\left[S^{(1)}, S^{(2)}\right]=0 .
$$

Since one can always add a constant to a nondegenerate potential, it follows that $1 / U$ defines an inverse Stäckel transform of $\widetilde{H}$ to $H$. We say that two quantum superintegrable systems are Stäckel equivalent if one can be obtained from the other by a Stäckel transform. We can now use Theorem 6 to carry over immediately the basic result for 2D Stäckel transforms of classical superintegrable systems to $2 \mathrm{D}$ quantum superintegrable systems. ${ }^{2}$

Theorem 7: Every nondegenerate second-order quantum superintegrable system in two variables is Stäckel equivalent to a superintegrable system on a constant curvature space.

\section{NONDEGENERATE 3D QUANTUM SYSTEMS}

Here we extend our analysis of classical 3D superintegrable systems with nondegenerate potentials to the quantum case. (This is less straightforward than in the 2D case.) As mentioned earlier, these systems arise only for functionally linearly independent bases of symmetries. For a manifold with metric $\mathrm{d} s^{2}=\lambda(x, y, z)\left(\mathrm{d} x^{2}+\mathrm{d} y^{2}+\mathrm{d} z^{2}\right)$ we replace the Hamiltonian $\mathcal{H}=\left(p_{1}^{2}+p_{2}^{2}\right.$ $\left.+p_{3}^{2}\right) / \lambda(x, y, z)+V(x, y, z)$ by a formally self-adjoint operator 


$$
\hat{H}=\frac{1}{\mu(x, y, z)} \sum_{k, j=1}^{3} \partial_{k}\left(\frac{\delta^{k j}}{\lambda(x, y, z)} \mu(x, y, z) \partial_{j}\right)+V(x, y, z)
$$

in local orthogonal coordinates. Here $\delta^{k j}$ is the Kronecker delta and the weight function $\mu$ is to be determined. Similarly, we replace a second-order symmetry of the Hamiltonian system $\mathcal{S}$ $=\sum_{k, j=1}^{3} a^{k j}(x, y, z) p_{k} p_{j}+W(x, y, z)$, with $a^{k j}=a^{j k}$, by the formally self-adjoint operator

$$
\hat{S}=\frac{1}{\mu} \sum_{k, j=1}^{3} \partial_{k}\left(a^{k j} \mu \partial_{j}\right)+W+\hat{W}, \quad a^{k j}=a^{j k},
$$

where the function $\hat{W}(x, y, z)$ is to be determined. These operators are formally self-adjoint with respect to the bilinear product

$$
\langle f, g\rangle=\int f(x, y, z) g(x, y, z) \mu(x, y, z) \mathrm{d} x \mathrm{~d} y \mathrm{~d} z
$$

on the manifold, i.e.,

$$
\langle f, \hat{H} g\rangle=\langle\hat{H} f, g\rangle, \quad\langle f, \hat{S} g\rangle=\langle\hat{S} f, g\rangle
$$

for all local $C^{\infty}$ functions $f, g$ with compact support on the manifold, where we set all boundary terms equal to 0 .

Now we assume that $\{\mathcal{H}, \mathcal{S}\}=0$ and require $[\hat{H}, \hat{S}]=0$. Since $\hat{H}, \hat{S}$ are formally self-adjoint, $[\hat{H}, \hat{S}]$ must be formally skew-adjoint. From our assumption $\{\mathcal{H}, \mathcal{S}\}=\mathbf{0}$ it is clear that the coefficients of the third-derivative terms $\partial_{i j k}$ in the commutator must vanish, hence also the coefficients of the second-order terms vanish. Thus there are functions $b_{i}$ such that

$$
[\hat{H}, \hat{S}]=\frac{1}{\mu} \sum_{i=1}^{3} \partial_{i}\left(b^{i} \mu\right)=\sum_{i=1}^{3}\left(b^{i} \partial_{i}+\frac{\left(b^{i} \mu\right)_{i}}{\mu}\right) .
$$

Using $\{\mathcal{H}, \mathcal{S}\}=0$, we see that

$$
b^{j}=\sum_{i=1}^{3}\left(\frac{1}{\lambda} \partial_{i i}+\frac{1}{\mu}\left(\frac{\mu}{\lambda}\right)_{i} \partial_{i}\right)\left(\frac{1}{\mu} \sum_{k=1}^{3}\left(a^{k j} \mu\right)_{k}\right)-\sum_{i, \ell=1}^{3}\left(a^{i \ell} \partial_{i \ell}+\frac{1}{\mu}\left(a^{i \ell} \mu\right)_{i} \partial_{\ell}\right)\left(\frac{1}{\mu}\left(\frac{\mu}{\lambda}\right)_{j}\right)+\frac{2}{\lambda} \hat{W}_{j} .
$$

This formula simplifies greatly if we choose $\mu=\lambda$. Indeed, we find

$$
b^{j}=-\frac{1}{\lambda} \partial_{j i k} a^{i k}+\frac{2}{\lambda} \hat{W}_{j}
$$

Here $i, j$, and $k$ are pairwise distinct. We can choose $\hat{W}_{j}=\frac{1}{2} \partial_{j i k} a^{i k}$, so that $b^{j} \equiv 0$ provided the integrability conditions

$$
\partial_{i i j k} a^{i k}=\partial_{i j j k} a^{j k}
$$

hold for $i, j, k$ pairwise distinct. These conditions are satisfied, as we can verify from the explicit expressions for second-order conformal Killing tensors contained in Ref. 3

Theorem 8:

$$
\{\mathcal{H}, \mathcal{S}\}=0 \Leftrightarrow[\hat{H}, \hat{S}]=0,
$$

where $\hat{H}, \hat{S}$ are given by (30) and (31) with $\mu=\lambda$ and $\hat{W}_{j}=\frac{1}{2} \partial_{j i k} a^{i k}$ (for $i, j, k$ pairwise distinct). 
We can follow a similar approach to find the quantum analogies of first-order symmetries $\mathcal{L}=\sum_{j=1}^{2} a^{j}(x, y, z) p_{j}$, by the formally skew-adjoint first-order operator (with respect to the bilinear product (32))

$$
\hat{L}=\frac{1}{\lambda} \sum_{j=1}^{3} \partial_{j}\left(a^{k j} \lambda\right)
$$

It is straightforward to prove the following result.

\section{Theorem 9:}

$$
\{\mathcal{H}, \mathcal{L}\}=0 \Leftrightarrow[\hat{H}, \hat{L}]=0,
$$

where $\hat{H}, \hat{L}$ are given by (30) and (33) with $\mu=\lambda$.

\section{THE SPACE OF THIRD-ORDER SYMMETRIES}

Now we investigate the third-order differential operators $K$ that commute with the Hamiltonian: $[H, K]=0$. The treatment for the conformally flat $3 \mathrm{D}$ case proceeds in almost exact analogy to the $2 \mathrm{D}$ case, so we just sketch the results.

1. Since the second-order symmetries are formally self-adjoint, the commutators will be skew-adjoint. Thus we can limit ourselves to $K$ that are skew adjoint.

2. Since $H$ encodes a four-parameter family of potentials, the symmetry $K$ must also be a function of the parameters. The highest order terms $a^{k j i} \partial_{k j i}$ in $K$ (symmetric in $k, j, i$ ) will be independent of the parameters but lower order terms may have linear parameter dependence.

3. The skew-adjoint requirement uniquely determines the coefficients of the second-order terms in $K$. They are

$$
\frac{3}{2 \lambda}\left(a^{k j i} \lambda\right)_{i} \partial_{k j}
$$

4. The skew-adjoint requirement means that there exist functions $a^{k j i}, \tilde{b}^{i}$ such that $K$ has the unique representation

$$
K=\sum_{k, j, i=1}^{3}\left(a^{k j i} \partial_{k j i}+\frac{3}{2 \lambda}\left(a^{k j i} \lambda\right)_{i} \partial_{k j}+\frac{1}{2 \lambda}\left(a^{k j i} \lambda\right)_{k j} \partial_{i}\right)+\sum_{i=1}^{3}\left(\tilde{b}^{i} \partial_{i}+\frac{1}{2 \lambda}\left(\tilde{b}^{i} \lambda\right)_{i}\right),
$$

where the functions $\tilde{b}^{i}(x, y, z)$ contain the parameter dependence.

5. Equating coefficients of the fourth-order terms in the operator condition $[H, K]=0$ where $K$ is given by (34) we obtain the classical requirements that the $a^{k j i}$ be the components of a thirdorder Killing tensor.

6. Equating coefficients of the third-order terms in the condition $[H, K]=0$ we obtain relations that are consequences of the Killing tensor requirements.

7. The remaining conditions on $K$ intertwine $\lambda, a^{k j i}, \tilde{b}^{i}$, and $V$, and are complicated. Rather than solve them directly, we make the unique decomposition

$$
\tilde{b}^{i}\left(x_{1}, x_{2}, x_{3}, V_{x_{1}}, V_{x_{2}}, V_{x_{3}}\right)=c^{i}\left(x_{1}, x_{2}, x_{3}\right)+b^{i}\left(x_{1}, x_{2}, x_{3}, V_{x_{1}}, V_{x_{2}}, V_{x_{3}}\right),
$$

where 


$$
b^{i}=\sum_{j=1}^{3} f^{\ell, j}\left(x_{1}, x_{2}, x_{3}\right) \frac{\partial V}{\partial x_{j}}\left(x_{1}, x_{2}, x_{3}\right),
$$

i.e., we can split off the parameter-dependent terms of $\widetilde{b}^{i}$ from the rest. Then, equating the linear parameter-dependent coefficients of the second-order terms in the symmetry operator condition, we obtain the conditions

$$
\begin{aligned}
& b_{k}^{j}+b_{j}^{k}=3 \lambda \sum_{s} a^{s k j} V_{s}, \quad j \neq k, \quad j, k=1,2,3, \\
& b_{j}^{j}=\frac{3}{2} \lambda \sum_{s} a^{s j j} V_{s}-\frac{1}{2} \sum_{s} b^{s}(\ln \lambda)_{s}, \quad j=1,2,3,
\end{aligned}
$$

identical to the classical requirement.

8. Equating the quadratic parameter-dependent coefficients of the zeroth-order terms in the symmetry operator condition, we obtain the requirement

$$
\sum_{s} b^{s} V_{s}=0
$$

identical to the classical equation. There can be at most one skew adjoint $K$ with given $a^{i j k}, b^{\ell}$.

Theorem 10: Let $K$ be a third-order skew-adjoint symmetry (11) for a superintegrable system with nondegenerate potential $V$ and $\tilde{b}^{i}=c^{i}(x, y, z)+b^{i}\left(x, y, z, V_{1}, V_{2}, V_{3}\right)$ where

$$
b^{i}=\sum_{j=1}^{3} f^{i, j}(x, y) \frac{\partial V}{\partial x_{j}}(x, y, z) .
$$

Then

$$
f^{\ell, j}+f^{j, \ell}=0, \quad 1 \leqslant \ell, j \leqslant 3
$$

and $K$ is uniquely determined by the four numbers

$$
f^{1,2}\left(x_{0}, y_{0}, z_{0}\right), \quad f^{1,3}\left(x_{0}, y_{0}, z_{0}\right), \quad f^{2,3}\left(x_{0}, y_{0}, z_{0}\right), \quad f_{3}^{1,2}\left(x_{0}, y_{0}, z_{0}\right)
$$

at any regular point $\left(x_{0}, y_{0}, z_{0}\right)$ of $V$.

Corollary 4: Let $V$ be a superintegrable nondegenerate potential. Then the space of thirdorder skew-adjoint symmetries is four-dimensional and is spanned by commutators of the secondorder self-adjoint symmetries.

In exact analogy with the classical case, we can use the standard form to prove multiseparabilty for conformally flat $3 \mathrm{D}$ quantum systems. ${ }^{4}$

Theorem 11: Let $V$ be a quantum superintegrable nondegenerate potential. Then the associated system is multiseparable.

\section{THE QUANTUM 3D QUADRATIC ALGEBRA}

We investigate the space of fourth-order differential operators $F$ that commute with the Hamiltonian: $[H, F]=0$. The treatment for the conformally flat $3 \mathrm{D}$ case proceeds in almost exact analogy to the $2 \mathrm{D}$ case, so we sketch the results.

1. We are interested in the space of fourth-order symmetries that is spanned by the double commutators $\left[\left[S^{(1)}, S^{(2)}\right], S^{(3)}\right]$ of second-order formally self-adjoint symmetries $S^{(j)}$ of the superintegrable system. The double commutators will be formally self-adjoint, so we can limit ourselves to $F$ that are self-adjoint. 
2. Since $H$ encodes a three-parameter family of potentials, the symmetry $F$ must also be a function of the parameters. The highest order terms $a^{\ell k j i} \partial_{k j i}$ in $F$ (symmetric in $\ell, k, j, i$ ) will be independent of the parameters but lower order terms may have linear or quadratic parameter dependence.

3. The self-adjoint requirement uniquely determines the third-order terms in $F$. They are

$$
\sum_{\ell, k, j, i} \frac{2}{\lambda}\left(a^{\ell k j i} \lambda\right)_{i} \partial_{\ell k j}
$$

4. The self-adjoint requirement means that there exist functions $a^{\ell k j i}, \tilde{b}^{i j}, \widetilde{W}$ such that $F$ has the unique representation

$$
F=\sum_{\ell, k, j, i=1}^{3} \frac{1}{\lambda} \partial_{i j}\left(a^{\ell k j i} \lambda \partial_{k \ell}\right)+\sum_{i, j=1}^{3} \frac{1}{\lambda} \partial_{i}\left(\tilde{b}^{i j} \lambda \partial_{j}\right)+\widetilde{W},
$$

where the functions $\tilde{b}^{i j}\left(x_{1}, x_{2}, x_{3}\right), \widetilde{W}\left(x_{1}, x_{2}, x_{3}\right)$ contain the parameter dependence.

5. Equating coefficients of the fifth-order terms in the operator condition $[H, F]=0$ we obtain the conditions for $a^{\ell k j i}$ to be a fourth-order Killing tensor.

6. The remaining conditions on $F$ intertwine $\lambda, a^{\ell k j i}, \tilde{b}^{j i}, \widetilde{W}$, and $V$, and are complicated. However, we can make the unique decomposition

$$
\tilde{b}^{j i}\left(x_{1}, x_{2}, x_{3}, V_{x_{1}}, V_{x_{2}}, V_{x_{3}}\right)=c^{j i}\left(x_{1}, x_{2}, x_{3}\right)+b^{j i}\left(x_{1}, x_{2}, x_{3}, V_{x_{1}}, V_{x_{2}}, V_{x_{3}}\right)
$$

where

$$
b^{j i}=\sum_{\alpha=1}^{4} f^{j i, \alpha} W^{(\alpha)}, \quad f^{j i, \alpha}=f^{i j, \alpha},
$$

and $W^{(j)}=V_{x_{j}}, W^{(4)}=V_{x_{1} x_{1}}$.

Then, equating the linear parameter-dependent terms of third order in the derivatives, and the quadratic parameter-dependent terms of first order in the derivatives, we obtain exactly the classical conditions on the $f^{j i, \alpha}$.

Since at most one self-adjoint $F$ can have data $a^{\ell k j i}, b^{k j}$, we find ${ }^{3}$

Theorem 12: The subspace of truly fourth-order self-adjoint symmetry operators is of dimension at most twenty-one.

If $A, B$ are linear operators, we define their symmetrized product by

$$
\{A, B\} \equiv \frac{1}{2}(A B+B A) .
$$

Theorem 13: The twenty-one distinct monomials $\left\{S^{(i j)}, S^{(j k)}\right\}$ form a basis for the space of fourth-order self-adjoint symmetry operators.

Using an approach very similar to the above we can easily show that the space of truly sixth-order formally self-adjoint operator symmetries of $H$ cannot exceed the classical maximal dimension of fifty-six. If $A, B, C$ are linear operators, we define their symmetrized product by

$$
\{A, B, C\} \equiv \frac{1}{6}(A B C+B A C+C A B+A C B+B C A+C B A) .
$$

Theorem 14: The fifty-six distinct standard monomials $\left\{S^{(h i)}, S^{(j k)}, S^{(\ell m)}\right\}$ form a basis for the space of sixth-order self-adjoint symmetry operators.

These theorems establish the closure of the quadratic algebra for 3D quantum superintegrable potentials: All fourth-order and sixth-order symmetry operators can be expressed as symmetric polynomials in the second-order symmetry operators. 


\section{COVARIANT FORMULATION FOR THE 3D QUANTUM CASE}

Theorem 8 yields an operator realization of the classical commutator brackets for secondorder symmetries but the differential operator part of $\hat{H}$, though formally self-adjoint with respect to the weight function $\lambda$, is not the Laplace-Beltrami operator on the manifold. We can obtain the Laplace-Beltrami operator, at the expense of altering the potential $V$, by means of an appropriate gauge transformation. We now turn to this construction.

Set

$$
H=e^{-\mathcal{R}} \hat{H} e^{\mathcal{R}}, \quad S=e^{-\mathcal{R}} \hat{S} e^{\mathcal{R}},
$$

where $\mathcal{R}(x, y, z)$ is a function to be determined. Then $[H, S]=0$ if and only if $[\hat{H}, \hat{S}]=0$. We will choose $\mathcal{R}$ such that the differential operator part of $H$ is the Laplace-Beltrami operator on the manifold with metric $\mathrm{d} s^{2}=\lambda\left(\mathrm{d} x^{2}+\mathrm{d} y^{2}+\mathrm{d} z^{2}\right)$.

It is straightforward to show that

$$
H=e^{-\mathcal{R}} \hat{H} e^{\mathcal{R}}=\frac{1}{\lambda} \sum_{i=1}^{3}\left(\partial_{i i}+2 \mathcal{R}_{i} \partial_{i}+\mathcal{R}_{i i}+\mathcal{R}_{i}^{2}\right)+V
$$

so, if we set $\mathcal{R}=1 / 4 \ln \lambda$, we have

$$
H=\sum_{i=1}^{3}\left(\frac{1}{\lambda^{3 / 2}} \partial_{i}\left(\lambda^{1 / 2} \partial_{i}\right)+\frac{\mathcal{R}_{i i}+\mathcal{R}_{i}^{2}}{\lambda}\right)+V .
$$

Similarly

$$
S=\sum_{i, j=1}^{3}\left(\frac{1}{\lambda^{3 / 2}} \partial_{i}\left(a^{i j} \lambda^{3 / 2} \partial_{j}\right)+a^{i j}\left(\mathcal{R}_{i j}+5 \mathcal{R}_{i} \mathcal{R}_{j}\right)+a_{i}^{i j} \mathcal{R}_{j}\right)+W+\hat{W} .
$$

The eigenvalue equation for $\hat{H}$ on the space with weight function $\mu=\lambda$ is $\hat{H} \Psi=E \Psi$. Setting $\Psi=e^{\mathcal{R}} \Phi=\lambda^{1 / 4} \Phi$ we see that the eigenvalue equation for $\Phi$ is $H \Phi=E \Phi$ and the eigenfunctions $\Phi$ lie in the space with weight function $\lambda^{3 / 2}$. Note that

$$
\sum_{i=1}^{3}\left(\mathcal{R}_{i i}+\mathcal{R}_{i}^{2}\right) / \lambda=-\frac{1}{8} R
$$

where $R$ is the Riemannian scalar curvature. The quantum potential is

$$
\widetilde{V}=-\frac{1}{8} R+V \text {. }
$$

If we supplement the classical symmetries with quantum adjustments the corresponding operators are

$$
\begin{gathered}
H=\frac{1}{\sqrt{g}} \partial_{i}\left(g^{i j} \sqrt{g} \partial_{j}\right)+\frac{1}{8} R, \\
S=\frac{1}{\sqrt{g}} \partial_{i}\left(a^{i j} \sqrt{g} \partial_{j}\right)+\frac{1}{16} a_{i}^{i} R-\frac{5}{16} a^{i j} R_{i j}-\frac{1}{16} \nabla_{i} \nabla_{j} a^{i j} .
\end{gathered}
$$

Here $\nabla_{j}$ is the usual covariant derivative on the Riemannian space. This formula always works, though $a^{i j}$ must be a Killing tensor for a conformally flat space, Indeed for a Hamiltonian $\mathcal{H}$ $=\lambda(x, y, z)\left(p_{x}^{2}+p_{y}^{2}+p_{z}^{2}\right)$ with symmetry $\mathcal{S}=a^{i j} p_{i} p_{j}$ the following conditions must be satisfied. If $a_{i j}$ is a Killing tensor for a conformally flat space with infinitesimal distance 


$$
\mathrm{d} s^{2}=e^{-J(x, y, z)}\left(\mathrm{d} x^{2}+\mathrm{d} y^{2}+\mathrm{d} z^{2}\right)
$$

then it must satisfy the equations

$$
\left.\partial_{\left({ }_{i j k}\right)}=g_{(i j} a_{k}\right)
$$

where

$$
\begin{gathered}
a_{1}=e^{2 J(x, y, z)}\left(a_{11} J_{x}-a_{12} J_{y}-a_{13} J_{z}\right), \quad a_{2}=e^{2 J(x, y, z)}\left(-a_{12} J_{x}+a_{22} J_{y}-a_{23} J_{z}\right), \\
a_{3}=e^{2 J(x, y, z)}\left(-a_{13} J_{x}-a_{23} J_{y}+a_{33} J_{z}\right) .
\end{gathered}
$$

Here (39) are the necessary and sufficient conditions that $a_{i j}$ is a conformal Killing tensor in flat space. We know all solutions for this set of equations. The only constraint is that there exist a function $J(x, y, z)$ such that the $a_{l}$ have the form indicated. Indeed, if we found the $a_{l}$ from the considerations of flat space it is clear that

$$
a_{k}=\frac{1}{5} \sum_{j}\left(\partial_{j} a_{j k}+\partial_{k} a_{j j}\right)
$$

These results carry over in a very satisfactory manner for superintegrable systems with nondegenerate potential. In this case the parameters occurring in the potential appear only in the $V$ and $W$ terms, exactly as before. The quantum corrections are independent of these parameters.

Theorem 15: Let $\mathcal{H}, \hat{H}$, and $H$ be defined as above where $\mathcal{H}$ defines a classical superintegrable system with nondegenerate potential $V$. Let $\mathcal{S}^{(1)}, \mathcal{S}^{(2)}$ be second-order symmetries of $\mathcal{H}$, with corresponding symmetry operators $\hat{S}^{(j)}, S^{(j)}$. Then

$$
\left\{\mathcal{S}^{(1)}, \mathcal{S}^{(2)}\right\}=0 \Leftrightarrow\left[\hat{S}^{(1)}, \hat{S}^{(2)}\right]=0 \Leftrightarrow\left[S^{(1)}, S^{(2)}\right]=0 .
$$

Corollary 5: Every conformally flat 3D classical superintegrable system with nondegenerate potential extends to a unique covariant quantum superintergrable system. The symmetries of the quantum system admit a quadratic algebra structure.

\section{THE STÄCKEL TRANSFORM FOR 3D QUANTUM SYSTEMS}

We work out the quantum analog of the Stäckel transform ${ }^{26,27}$ for classical systems. Suppose we have a superintegrable system with Schrödinger operator

$$
H=\frac{1}{\lambda^{3 / 2}(x, y, z)} \sum_{i=1}^{3} \partial_{i}\left(\lambda^{1 / 2}(x, y, z) \partial_{i}\right)-\frac{1}{8} R_{\lambda}(x, y, z)+V(x, y, z)
$$

in local orthogonal coordinates, with scalar curvature $R_{\lambda}$ and nondegenerate potential $V(x, y, z)$ :

$$
\begin{gathered}
V_{33}=V_{11}+A^{33} V_{1}+B^{33} V_{2}+C^{33} V_{3}, \\
V_{22}=V_{11}+A^{22} V_{1}+B^{22} V_{2}+C^{22} V_{3}, \\
V_{23}=A^{23} V_{1}+B^{23} V_{2}+C^{23} V_{3}, \\
V_{13}=A^{13} V_{1}+B^{13} V_{2}+C^{13} V_{3}, \\
V_{12}=A^{12} V_{1}+B^{12} V_{2}+C^{12} V_{3}
\end{gathered}
$$

and suppose $U(x, y, z)$ is a particular solution of Eq. (41), nonzero in an open set. Then the transformed system 


$$
\widetilde{H}=H=\frac{1}{\widetilde{\lambda}^{3 / 2}(x, y, z)} \sum_{i=1}^{3} \partial_{i}\left(\tilde{\lambda}^{1 / 2}(x, y, z) \partial_{i}\right)-\frac{1}{8} R_{\lambda}^{\sim}(x, y, z)+\tilde{V}(x, y, z)
$$

with nondegenerate potential $\tilde{V}(x, y, z)$ :

$$
\begin{aligned}
& \widetilde{V}_{33}=\widetilde{V}_{11}+\widetilde{A}^{33} \widetilde{V}_{1}+\widetilde{B}^{33} \widetilde{V}_{2}+\widetilde{C}^{33} \widetilde{V}_{3}, \\
& \tilde{V}_{22}=\widetilde{V}_{11}+\widetilde{A}^{22} \tilde{V}_{1}+\widetilde{B}^{22} \widetilde{V}_{2}+\widetilde{C}^{22} \widetilde{V}_{3}, \\
& \tilde{V}_{23}=\widetilde{A}^{23} \tilde{V}_{1}+\widetilde{B}^{23} \tilde{V}_{2}+\widetilde{C}^{23} \tilde{V}_{3}, \\
& \widetilde{V}_{13}=\widetilde{A}^{13} \widetilde{V}_{1}+\widetilde{B}^{13} \tilde{V}_{2}+\widetilde{C}^{13} \widetilde{V}_{3}, \\
& \widetilde{V}_{12}=\widetilde{A}^{12} \widetilde{V}_{1}+\widetilde{B}^{12} \widetilde{V}_{2}+\widetilde{C}^{12} \widetilde{V}_{3},
\end{aligned}
$$

is also superintegrable, where

$$
\begin{gathered}
\tilde{\lambda}=\lambda U, \quad \tilde{V}=\frac{V}{U}, \\
\tilde{A}^{33}=A^{33}+2 \frac{U_{1}}{U}, \quad \tilde{B}^{33}=B^{33}, \quad \tilde{C}^{33}=C^{33}-2 \frac{U_{3}}{U}, \\
\widetilde{A}^{22}=A^{22}+2 \frac{U_{1}}{U}, \quad \widetilde{B}^{22}=B^{22}-2 \frac{U_{2}}{U}, \quad \tilde{C}^{22}=C^{22}, \\
\widetilde{A}^{23}=A^{23}, \quad \widetilde{B}^{23}=B^{23}-\frac{U_{3}}{U}, \quad \tilde{C}^{23}=C^{23}-\frac{U_{2}}{U}, \\
\widetilde{A}^{13}=A^{13}-\frac{U_{3}}{U}, \quad \widetilde{B}^{13}=B^{13}, \quad \widetilde{C}^{13}=C^{13}-\frac{U_{1}}{U}, \\
\widetilde{A}^{12}=A^{12}-\frac{U_{2}}{U}, \quad \widetilde{B}^{12}=B^{12}-\frac{U_{1}}{U}, \quad \tilde{C}^{12}=C^{12} .
\end{gathered}
$$

Indeed, let $S=\sum\left(1 / \lambda^{3 / 2}\right) \partial_{i}\left(a^{i j} \lambda^{3 / 2} \partial_{j}\right)+\mathcal{W}_{R}+W=S_{0}+\mathcal{W}_{R}+W$ be a second-order formally selfadjoint symmetry operator of $H$, where $\mathcal{W}_{R}$ is the potential term that depends on the curvature $R$ and $W$ is the part that depends on $V$. Let $S_{U}=\Sigma\left(1 / \lambda^{3 / 2}\right) \partial_{i}\left(a^{i j} \lambda^{3 / 2} \partial_{j}\right)+\mathcal{W}_{R}+W_{U}=S_{0}+\mathcal{W}_{R}+W_{U}$ be the special case of this that is in involution with

$$
\frac{1}{\lambda^{3 / 2}} \sum_{i=1}^{3} \partial_{i}\left(\lambda^{1 / 2} \partial_{i}\right)-\frac{1}{8} R_{\lambda}+U
$$

Then 


$$
\tilde{S}=S_{0}-\frac{W_{U}}{U} H+\frac{1}{U} H
$$

is the corresponding formally self-adjoint symmetry operator of $\widetilde{H}$, with respect to the metric $d \widetilde{s}^{2}=\lambda U\left(\mathrm{~d} x^{2}+\mathrm{d} y^{2}+\mathrm{d} z^{2}\right)$.

\section{Theorem 16:}

1.

$$
[\tilde{H}, \tilde{S}]=0 \Leftrightarrow[H, S]=0 .
$$

2.

$$
\tilde{S}=\sum_{i j} \frac{1}{(\lambda U)^{3 / 2}} \partial_{i}\left(\left(a^{i j}+\delta^{i j} \frac{1-W_{U}}{\lambda U}\right)(\lambda U)^{3 / 2}\right) \partial_{j}+\left(\mathcal{W}_{R}+\left(\frac{W_{U}}{U}-\frac{1}{U}\right) \frac{R_{\lambda}}{8}+\left(W-\frac{W_{U} V}{U}+\frac{V}{U}\right) .\right.
$$

Proof:

1. We perform an inverse gauge transformation on $H, S$ to return them to the forms $\hat{H}, \hat{S}$, (30) and (31), with $\mu=\lambda$ and $\hat{W}_{j}=\frac{1}{2} \partial_{j i k} a^{i k}$ (for $i, j, k$ pairwise distinct). Similarly we perform an inverse gauge transformation on $\widetilde{H}, \tilde{S}$ to return them to the forms $\hat{\widetilde{H}}, \hat{\widetilde{S}},(30)$ and (31), with $\mu=U \lambda$. These commuting operators are formally self-adjoint with respect to the weight function $U \lambda$. Then it is a straightforward computation to verify that $[\hat{\widetilde{H}}, \hat{\tilde{S}}]=0 \Leftrightarrow[\hat{H}, \hat{S}]=0$. Indeed, just as in the $2 \mathrm{D}$ case, one needs only the identities

$$
\left[\hat{H}_{0}, \hat{S}_{0}\right]=0, \quad\left[\hat{H}_{0}+V, \hat{S}_{0}+W\right]=0, \quad\left[\hat{H}_{0}+U, \hat{S}_{0}+W_{U}\right]=0
$$

and

$$
[A, B C]=B[A, C]+[A, B] C, \quad\left[A, \frac{1}{U}\right]=-\frac{1}{U}[A, U] \frac{1}{U}
$$

for linear operators $A, B, C$ and nonzero function $U$. Then the first part of the theorem follows from applying the original gauge transformations to take $\hat{H}, \hat{S}$ to $H, S$ and $\hat{\widetilde{H}}, \hat{\tilde{S}}$ to $\widetilde{H}, \tilde{S}$.

2. This follows from the fact that $\partial_{i} W_{U}=\lambda \Sigma_{j} a^{i j} U_{j}$.

Corollary 6: If $S^{(1)}, S^{(2)}$ are second-order symmetry operators for $H$, then

Q.E.D.

$$
\left[\widetilde{S}^{(1)}, \widetilde{S}^{(2)}\right]=0 \Leftrightarrow\left[S^{(1)}, S^{(2)}\right]=0 .
$$

At this point it is clear that the basic classical result for 3D Stäckel transforms of conformally flat classical superintegrable systems contained in Ref. 4 can be carried over to 3D quantum superintegrable systems.

Theorem 17: Every nondegenerate second-order quantum superintegrable system on a $3 D$ conformally flat space is Stäckel equivalent to a superintegrable system on a constant curvature space.

\section{CONCLUSIONS AND OUTLOOK}

We showed that 2D classical second-order superintegrable systems with nondegenerate potential and the corresponding 3D conformally flat systems each have a unique quantum superintegrable extension, and that the closure of the quadratic algebra and basic structure theory is unchanged at the quantum level. A critical feature of the proofs is use of the formal self-adjoint and skew-adjoint properties of the higher order symmetry operators. For the 2D case the extension is completely straightforward and the quantum extension has the same nondegenerate potential as the classical system. For the 3D systems a two-step procedure is required. First the classical 
system is extended to a quantum system with appropriate formal self and skew adjoint symmetries and such that the potential remains unchanged. This quantum system, however, is not covariant, i.e., the Schrödinger operator does not correspond to a Laplace-Beltrami operator on a curved manifold. The second step in the procedure is to perform a gauge transformation to obtain covariantly correct Schrödinger operators. This alters the potential by adding a term that depends on the scalar curvature. We also showed that the Stäckel transform has a unique quantum extension and it remains true that all of our quantum superintegrable systems are Stäckel transforms of constant curvature superintegrable systems.

All 2D systems have been classified and we are making considerable progress on the 3D classification theory for systems with functionally linearly independent bases of symmetries, ${ }^{4}$ though the problem is complicated. The next steps in our program are (1) to study 3D superintegrable systems with degenerate potentials and (2) to study nondegenerate superintegrable systems in higher dimensions.

\section{ACKNOWLEDGMENTS}

A referee's suggestion that we show how our structure theory relates to the generalized Calogero potential enabled us to clarify this relation and to point out the importance of the concept of functional linear independence for functionally independent symmetries. In the process we found and corrected an error in a paper (Ref. 3) of the series.

${ }^{1}$ E. G. Kalnins, J. M. Kress, and W. Miller, Jr., J. Math. Phys. 46, 053509 (2005).

${ }^{2}$ E. G. Kalnins, J. M. Kress, and W. Miller, Jr., J. Math. Phys. 46, 053510 (2005).

${ }^{3}$ E. G. Kalnins, J. M. Kress, and W. Miller, Jr., J. Math. Phys. 46, 103507 (2005).

${ }^{4}$ E. G. Kalnins, J. M. Kress, and W. Miller, Jr., J. Math. Phys. 47, 043514 (2006).

${ }^{5}$ E. G. Kalnins, W. Miller, Jr., and G. S. Pogosyan, J. Math. Phys. 47, 033502 (2006).

${ }^{6}$ S. Rauch-Wojciechowski, Phys. Lett. 95A, 279 (1983).

${ }^{7}$ N. W. Evans, Phys. Rev. A 41, 5666 (1990); J. Math. Phys. 32, 3369 (1991).

${ }^{8}$ J. Friš, V. Mandrosov, Ya. A. Smorodinsky, M. Uhlír, and P. Winternitz, Phys. Lett. 16, 354 (1965).

${ }^{9}$ J. Friš, Ya. A. Smorodinskii, M. Uhlír, and P. Winternitz, Sov. J. Nucl. Phys. 4, 444 (1967).

${ }^{10}$ A. A. Makarov, Ya. A. Smorodinsky, Kh. Valiev, and P. Winternitz, Nuovo Cimento A 52, 1061 (1967).

${ }^{11}$ F. Calogero, J. Math. Phys. 10, 2191 (1969).

${ }^{12}$ L. P. Eisenhart, Riemannian Geometry (Princeton University Press, Princeton, NJ, 1949).

${ }^{13}$ W. Miller, Jr., Symmetry and Separation of Variables (Addison-Wesley, Providence, RI, 1977).

${ }^{14}$ E. G. Kalnins and W. Miller, Jr., SIAM J. Math. Anal. 11, 1011 (1980).

${ }^{15}$ W. Miller, Jr., Proceedings of School and Workshop on Nonlinear Phenomena, Oaxtepec, Mexico, November 29December 17, 1982, Lecture Notes in Physics Vol. 189 (Springer, New York, 1983).

${ }^{16}$ E. G. Kalnins, Separation of Variables for Riemannian Spaces of Constant Curvature, Pitman Monographs and Surveys in Pure and Applied Mathematics Vol. 28 (Longman, Essex, 1986), pp. 184-208.

${ }^{17}$ W. Miller, Jr., in Symmetries and Non-linear Phenomena (World Scientific, Singapore, 1988), pp. 188-221.

${ }^{18}$ C. Daskaloyannis and K. Ypsilantis, J. Math. Phys. 47, 042904 (2006).

${ }^{19}$ E. G. Kalnins, W. Miller, Jr., and G. S. Pogosyan, J. Math. Phys. 37, 6439 (1996).

${ }^{20}$ D. Bonatos, C. Daskaloyannis, and K. Kokkotas, Phys. Rev. A 50, 3700 (1994).

${ }^{21}$ C. Daskaloyannis, J. Math. Phys. 42, 1100 (2001).

${ }^{22}$ S. P. Smith, Trans. Am. Math. Soc. 322, 285 (1990).

${ }^{23}$ F. Calogero, J. Math. Phys. 12, 419 (1971).

${ }^{24}$ S. Rauch-Wojciechowski and C. Waksjö, J. Nonlinear Math. Phys. 12, 535 (2005).

${ }^{25}$ J. T. Horwood, R. G. McLenaghan, and R. G. Smirnov, Commun. Math. Phys. 259, 679 (2005).

${ }^{26}$ C. P. Boyer, E. G. Kalnins, and W. Miller, Jr., SIAM J. Math. Anal. 17, 778 (1986).

${ }^{27}$ J. Hietarinta, B. Grammaticos, B. Dorizzi, and A. Ramani, Phys. Rev. Lett. 53, 1707 (1984). 